Chapman University

Chapman University Digital Commons

ESI Working Papers

Economic Science Institute

3-7-2018

\title{
Your Money or Your Time? Experimental Evidence on Overbidding in All-Pay Auctions
}

\author{
Adriana Breaban \\ ChapmanUniversity, breaban@chapman.edu \\ Charles N. Noussair \\ University of Arizona \\ Andreea Victoria Popescu \\ Tilburg University
}

Follow this and additional works at: https://digitalcommons.chapman.edu/esi_working_papers

Part of the Econometrics Commons, Economic Theory Commons, and the Other Economics Commons

\section{Recommended Citation}

Breaban, A., Noussair, C.N., \& Popescu, A.V. (2018). Your money or your time? Experimental evidence on overbidding in all-pay auctions. ESI Working Paper 18-20. Retrieved from https://digitalcommons.chapman.edu/esi_working_papers/263/

This Article is brought to you for free and open access by the Economic Science Institute at Chapman University Digital Commons. It has been accepted for inclusion in ESI Working Papers by an authorized administrator of Chapman University Digital Commons. For more information, please contactlaughtin@chapman.edu. 


\title{
Your Money or Your Time? Experimental Evidence on Overbidding in All-Pay Auctions
}

\author{
Comments \\ Working Paper 18-20
}




\title{
Your money or your time? Experimental evidence on overbidding in all-pay auctions
}

Adriana Breaban ${ }^{1}$

\author{
Charles N. Noussair
}

Andreea Victoria Popescu

\author{
March 7, 2018
}

\begin{abstract}
Competition for a prize frequently takes the form of dedicating time toward winning a contest. Those who spend the most time become more likely to obtain the prize. We model this competition as an all-pay auction under incomplete information, and report an experiment in which expenditures and rewards are in terms of time. In the experiment, subjects must stay in the laboratory doing nothing for an initially prespecified length of time. However, they can bid, in terms of time, to leave early. The auction has an allpay structure so that if an individual does not submit the highest bid within her group, she must stay for the additional time that she bid. We correlate behavior in this game with behavior in an isomorphic all-pay auction played with money bids. We also consider how two measures of sophistication, the Cognitive Reflection Test (CRT) score, and performance on a probability calibration task, correlate with behavior. We find strong similarities in overall behavior between the auctions conducted with money and with time. Bidding greater than equilibrium levels is typical, and as a consequence, average earnings are negative in both auctions. Thus, the result that there is overdissipation of rent in all-pay auctions extends to competition in terms of time. Higher CRT score and more accurate probability calibration correlate with better decisions in auctions played for money but not those played for time.
\end{abstract}

\section{Introduction}

Competition for prizes is a basic feature of many economic and social interactions. Firms compete to develop new products, athletes vie to win medals and cash prizes, students compete for grades, and applicants fight for jobs. Economists, beginning with Tullock (1980) have modeled these competitions as rent seeking games, in which competitors expend resources, with greater expenditures translating into a higher probability of winning the prize. An extreme case is winnertake-all competition, which can be modeled as an all-pay auction. In an all-pay auction, each of a number of competing bidders makes a bid. This bid is typically interpreted as an expenditure of money, time or effort. The player making the highest bid wins the prize, but losing players pay the

\footnotetext{
${ }^{1}$ Breaban, Economic Science Institute, Chapman University. Noussair: Department of Economics and Economic Science Laboratory, University of Arizona. Popescu, Department of Economics, Tilburg University. Correspondence to Charles Noussair at cnoussair@email.arizona.edu.
} 
amount that they bid. In a first-price all-pay auction, the winning bidder also pays an amount equal to her own bid. ${ }^{2}$ Therefore, everyone participating in the game incurs a cost, making the setting well-suited to studying competition.

However, money is not the only medium of reward used in competitions. In this paper, we study competition in terms of time. In many settings, the time spent on a task translates into an advantage. The popular author Malcolm Gladwell (2008) captures the essence of this correlation with his remark that becoming a world-class expert in a domain requires 10000 hours of practice. Indeed, in many of the examples cited in the first paragraph, from competition to develop a new product, to achievement in work, school, music or athletics, the time one commits to a task is critical to achieving a high likelihood of success. This raises an important question: do individuals maintain the same strategies across different reward domains when it comes to competing in contests? This paper focuses on this question.

The theory of auctions is general in the sense that the valuations and bids for items may be thought of in terms of money, effort or time, and the underlying logic of the models is the same regardless of the medium. However, the experimental research that has been conducted to date on all-pay auctions has always employed bidding in terms of money, which is the standard medium of reward in experimental studies in economics. Thus, bidding behavior in terms of money is the only setting that is well-understood. This literature has for the most part documented bidding that is more aggressive than equilibrium levels (Davis and Reilly, 1998; Potters et al., 1998³ Gneezy and Smorodinsky, 2006; Lugovskyy et al., 2010), and overdissipation of the available rent, in settings with complete information. Barut et al. (2002), Kaplan et al. (2002), Noussair and Silver (2006), and Hyndeman et al. (2012) also observe overbidding relative to equilibrium in environments with incomplete information. See Decheneaux et al. (2015) for a review of the literature.

Understanding the allocation of time is also of more general interest, because individuals are required to make decisions regarding how to spend one's time on a continual basis. The total amount of time available to an individual is fixed. Consequently, agents have to constantly evaluate the benefits and costs of different time allocations. Despite being of tremendous interest,

\footnotetext{
${ }^{2}$ There are a number of other variations, including a second price all-pay auction, in which the winner pays an amount equal to the second highest bid, and a dynamic version of the second price all-pay auction, known as the war of attrition (Maynard Smith, 1974).

${ }^{3}$ This paper is an exception. Potters et al (1998) find that average bids are close to the Nash equilibrium level.
} 
only a limited number of studies in the social sciences have employed time as a source of value. This strand of research corroborates the idea that the behavior of players does not change when the medium of reward changes. Berger et al. (2012) observe no significant differences between ultimatum games where players bargain over money or time. Noussair and Stoop (2015) also find that agents do not change their pattern of behavior significantly in either dictator, ultimatum, or trust games when playing in terms of money rather than time. No previous experimental studies, of which we are aware, have studied the behavior of individuals in an all-pay auction in terms of time.

In the experiment reported here, we study whether participants' bidding behavior exhibits similar patterns when the effort exerted is in terms of time rather than money. To test this hypothesis, we create two different protocols. In the first protocol, called MT, subjects participate first in fifteen monetary all-pay auctions, and play a one shot all-pay auction in terms of time thereafter. Under the second protocol, called T, participants play a one-shot time auction only.

Our environment is one of incomplete information, specifically the independent private value structure introduced by Vickrey (1961). When playing in terms of money, individuals have a private monetary value for winning the item, drawn from a distribution that is common knowledge and the same for all bidders. Players then engage in a first-price all-pay auction. They submit monetary bids simultaneously. The highest bidder receives the item and every bidder pays what she bid, regardless of whether or not she won the auction.

When the auction is played in terms of time, subjects begin with an obligation to stay in a private, fully-enclosed cubicle for 30 minutes. During their waiting time, they are not permitted to engage in any activity except for sitting quietly. They receive a valuation, denominated in terms of minutes, for winning the auction. This valuation entitles them to leave the experiment early, by the number of minutes indicated in their valuation, if they win the auction. Each player then simultaneously submits a bid in terms of time. A bid consists of a commitment to stay a number of minutes equal to their bid, in addition to the initial baseline of 30 minutes. The player who bids the highest receives a reduction in his required time in the lab by a number of minutes equal to her valuation minus her bid. All other players are required to stay an additional number of minutes equal to their bids.

We first compare bidding with equilibrium behavior, and study overall auction revenue and allocative efficiency. We then consider the relationship between cognitive ability and behavior in 
the auctions. We study whether bidders who display greater ability bid closer to the equilibrium levels and achieve greater earnings than other players. The Cognitive Reflection test, proposed by Frederick (2005), is employed to measure this relationship. The test has been found to correlate strongly with behavior and earnings in second-price auctions (Schneider and Porter, 2016) and asset markets (Corgnet et al., 2014; Breaban and Noussair, 2015; Bosch-Rosa et al., 2017).

Inaccurate beliefs about the relationship between a bid level and the probability that the bid is higher than the bids of all other players, might also account for overbidding. While overbidding in first-price, winner-pay auctions is consistent with risk aversion (Cox et al., 1982, 1988), Goeree et al. (2002) suggest that overbidding might result from participants' misperception of the probability of winning the auction. Armantier and Treich (2009) present evidence in support of the claim that underestimation of probabilities leads to overbidding in first-price, winner-pay auctions. Baharad and Nitzan (2008) model how the distortion of probabilistic events, encapsulated by prospect theory, can give rise to overbidding in contests. In our experiment, we implement a measurement protocol for probability calibration in auctions, and correlate the scores of individuals with their earnings and their deviations from equilibrium bidding.

Our results show that the general patterns in aggregate behavior are similar in auctions for money and for time. Regardless of the reward medium, bidding is on average higher than equilibrium levels and players lose money on average. The available rent is more than fully dissipated for both money and time. However, cognitive reflection test scores and probability calibration are positively related to earnings only in the money auction, and not in the time auction. A gender effect appears in the time auction in that women bid more aggressively than men. Thus, while the general pattern of overbidding relative to equilibrium is robust to the change in reward medium, there appear to be some differences between the way bidders approach competition over money and over time.

The paper proceeds as follows. In section 2, the symmetric equilibrium bidding strategy of an all-pay auction is presented for both the monetary and the time auctions. The experimental design is described in section 3. Section 4 presents an analysis of the experimental results for the money auction. Section 5 reports the results of the time auctions, and section 6 contrasts the two reward media. Section 7 contains some concluding remarks. 


\section{Model}

In this section, we derive a Bayesian equilibrium for the auctions that we study in our experiment. This equilibrium serves as the benchmark against which we compare the experimental data. Section 2.1 does so for the monetary auction, following closely the arguments of Noussair and Silver (2006), and Section 2.2 presents a similar analysis for the time auction.

\subsection{Single unit, first price, all-pay auctions for money}

Consider an all pay auction for one item that is to be sold to the highest bidder among $n$ competitors. Each bidder $i=\{1, \ldots, n\}$ draws a valuation for the item, $v_{i}$, independently from a uniform distribution on $[0, \bar{v}]$. Valuations are private information, but the number of players and the distribution of valuations is common knowledge.

The auction follows first price all-pay rules. Each bidder $i$ submits a bid $b_{i} \geq 0$ simultaneously. The person who submits the highest bid wins the item for sale. All bidders pay their bid, regardless of whether or not they obtain the item.

The utility of player $i$ then takes the following form:

$$
\Pi_{i}\left(b_{i}, v_{i}\right)=\left\{\begin{array}{lr}
v_{i}-b_{i} & \text { if } b_{i}>b_{j} \forall j \neq i \\
-b_{i} & \text { if } \exists j \neq i \text { such that } b_{i}<b_{j}
\end{array}\right.
$$

The first term, $v_{i}-b_{i}$, is the bidder's payoff in the event that she wins the auction. This event occurs if $i$ submits the highest bid among all players, or in other words, if $b_{i}>b_{j} \forall j \neq i$. Each bidder $i$ receives $v_{i}$ in value from the item and pays $b_{i}$ to acquire it. The second expression, $-b_{i}$, is the payoff of bidder $i$ in the event that she is not the highest bidder, in which case she pays her bid. This outcome occurs if $\exists j \neq i$ such that $b_{i}<b_{j}$.

Suppose that all bidders are using a symmetric Bayesian equilibrium bidding strategy $\beta(v)$, and further assume that the equilibrium bidding strategy is monotonic so that $\beta^{\prime}(v)>0$. There is a symmetric equilibrium bidding function $\beta\left(v_{i}\right)$, which in the case of five bidders has the form

$$
\beta\left(v_{i}\right)=\frac{\bar{v}(n-1)}{n}\left(\frac{v_{i}}{\bar{v}}\right)^{n} \text {. }
$$

For the parameters of our experiment, $n=5$, and $\bar{v}=1000$, equation (2) becomes

$$
\beta\left(v_{i}\right)=800 *\left(\frac{v_{i}}{1000}\right)^{5} .
$$

The equilibrium expected payoff for bidder $i$ is:

$$
\Pi\left(v_{i}\right)=\left(\frac{1}{\bar{v}}\right)^{n-1} * v_{i}^{n} * \frac{1}{n} .
$$


For our parameters, the expected payoff is:

$$
\Pi\left(v_{i}\right)=\left(\frac{1}{1000}\right)^{4} * v_{i}^{5} * \frac{1}{5}=200 *\left(\frac{v_{i}}{1000}\right)^{5} .
$$

A detailed derivation is given in the appendix. The argument parallels that provided by Noussair and Silver (2006).

\subsection{All-pay auction with time as a medium of reward and payment}

Now consider an all-pay auction with time as a medium of reward rather than money. Before the auction takes place, each bidder has a commitment of time to the auctioneer, equal to $K$ minutes. The activity during these $K$ minutes has a marginal disutility, which is assumed to be constant over time. ${ }^{4}$ In the experiment, this consists of waiting for $K$ minutes in the laboratory. The valuation, $v_{i}$, drawn by each participant, represents the number of minutes that can be subtracted from the mandatory $K$ minutes (allowing the participant to leave the experiment earlier) if bidder $i$ wins the auction.

Each bidder simultaneously submits a positive sealed bid $b_{i}$, representing the number of minutes that she offers to remain in the laboratory, on top of the initial time $K$. The utility of player $i$ is the following:

$U_{i}\left(b_{i}, v_{i}\right)=\left\{\begin{array}{lr}v_{i}-b_{i}-K & \text { if } b_{i}>b_{j} \forall i \neq j \\ -b_{i}-K & \text { if } \exists j \neq i \text { such that } b_{i}<b_{j}\end{array}\right.$

Since $K$ is constant, it does not enter the maximization problem. As a result, the equilibrium bidding function is analogous to the case where the medium of effort is monetary:

$$
\beta\left(v_{i}\right)=\left(\frac{1}{\bar{v}}\right)^{n-1} * \frac{n-1}{n} * v_{i}^{n}
$$

For the parameters used in the experiment, where there are 5 bidders, whose valuations are drawn from $[0,30]$, the equilibrium bidding function is:

$$
\beta\left(v_{i}\right)=\left(\frac{1}{30}\right)^{4} * \frac{4}{5} * v_{i}^{5}
$$

The maximum possible bid consistent with the risk neutral Bayesian equilibrium, is $\beta(30)=24$.

\footnotetext{
${ }^{4}$ This constant marginal disutility can be thought of as risk neutrality in time. It means that an individual is indifferent between waiting a fixed amount of time and a random amount of time with the same expected length.
} 


\section{The experiment}

\subsection{General description}

The sessions were conducted at the CentER experimental laboratory, at Tilburg University, The Netherlands, in 2013 and 2016. Participants were Bachelor's and Master's students at the University. The experiment had two protocols, called MT and T, and was designed to allow both within-and between-subject comparisons of auctions conducted for money and time. In each session, only one protocol was in effect. MT consisted of 15 monetary auctions, followed by one time auction, while $\mathrm{T}$ consisted of one time auction only. Each individual participated in only one session. In both treatments, subjects also completed tasks to measure their cognitive ability and probability calibration, before the auctions took place.

In total, 12 experimental sessions were conducted, six under each protocol. Ten participants were present in each session, and thus there were two auctions with five bidders conducted simultaneously in each period. On average, each session under the MT protocol lasted approximately 2 hours and 20 minutes and subjects earned an average of $21.7 €^{5}$. Each session in the $\mathrm{T}$ protocol lasted approximately 1 hour and 10 minutes and participants earned on average 13.4 $€$. Subjects received their earnings by electronic cash transfer made on the evening of the same day as the experiment.

The experiment was conducted using pen and paper, and thus was not computerized. At the beginning of the session, the experimenter distributed handouts containing the instructions for the experiment. The instructions were read aloud by the experimenter before each part of the experiment. Subjects were seated in individual private fully enclosed cubicles that ensured that no communication between individuals could occur during the experiment. All questions were answered privately.

\footnotetext{
${ }^{5}$ On average, in the MT treatment, each participant earned $1.95 €$ in the CRT task, $7.35 €$ in the probability calibration task, $-0.89 €$ during the monetary all-pay auctions, plus a $16 €$ show-up fee. If losses were incurred during the monetary auctions above the initial cash balance of 1000ECU, the loss would be deducted from total earnings made in other parts of the experiment. Subjects were informed of this rule at the beginning of the monetary auction. Total earnings were sent via bank transfer within 48 hours of the end of the session. Payment via bank transfer is a standard procedure at Tilburg University and was how subjects expected to be paid.
} 


\subsection{The monetary auction}

Fifteen periods of monetary auctions were conducted in the MT protocol. At the beginning of period 1, subjects received an initial cash balance of 1000 ECU (Experimental Currency Units). The experimental currency was converted to Euros at a rate of 100 ECU per 1 Euro. New valuations for the item, denominated in ECU, were drawn each period, from a discrete uniform distribution on $[1,1000]$. Draws were independent across bidders and from one period to the next. Bids were made in terms of ECU and had to be greater than or equal to 0.

In each of the 15 periods, bidders submitted bids for a fictitious object available for sale. They wrote their bids down on a form provided to them, which was then collected by the experimenter. All bids were submitted simultaneously in the sense that no subject could observe the choice of any other player before she submitted her bid. The instructions and forms that subjects filled out are provided in the Appendix.

After collecting the forms and noting the winner, the experimenter returned to inform each bidder separately whether she was successful in winning the auction. The bidder with the highest bid won the auction and received her valuation minus her bid as a payoff for the period. All other subjects incurred a loss equal to the amount of their bid. The participants who did not win the auction did not learn the winning bid. In case of a tie, the winner was chosen by a coin flip. There was one practice period before the fifteen periods which counted toward subjects' earnings.

\subsection{The time auction}

One time auction took place in both the MT and the T sessions. In the time auction, subjects were asked to remain in their cubicles for 30 minutes, during which time they were required to be passive. They were not allowed to use any electronic device, read, write, or communicate with other participants ${ }^{6}$. They received an additional $6 €$ for the time auction in addition to any earnings from other tasks. Subjects were given the opportunity to participate in one all-pay auction in which, if successful, they could decrease their waiting time in the laboratory from the initial 30

\footnotetext{
${ }^{6}$ This ensured that no other utility was derived during the waiting time, as would have been the case if participants could have used their phones or laptops, for instance.
} 
minutes and leave the experiment early. Therefore, the currency used within this auction was time (in minutes).

Each participant received a "Time exemption", the term used to describe her valuation. The time exemption indicated the number of minutes that she could leave the session early if she won the auction. The time exemption for each individual was drawn independently from a discrete uniform distribution ${ }^{7}$ on $[0,30]$ which was common knowledge. Players then submitted their bids in terms of minutes. The participant who bid the highest had her time exemption subtracted from the initial 30 minutes, and the rest then had their bid added to the 30-minute baseline waiting time. That is, the winner could leave the laboratory after 30 minutes minus her time exemption plus the number of minutes that she bid. All other bidders had to stay for 30 minutes plus the number of minutes that they bid. The instructions and examples of the time auction were read aloud. They were similar to the monetary all-pay auction, but adjusted to fit the different reward medium and valuation distribution. The instructions can be found in the appendix.

A practice period was held, in which subjects did not actually have to wait. This was included to familiarize subjects with the process. This was followed by a one-shot game, which did count. After the participants placed their bids, they were informed of whether they won the auction or not, and the total amount of time they were required to wait in their cubicle. When the waiting time for an individual expired, the experimenter notified the person that she could leave the experiment. Under both treatments, after the auctions were played, a questionnaire regarding background information was given to each participant before their waiting period began.

\subsection{Probability Calibration}

Under both the MT and T protocols, we elicited a measure of how well subjects were calibrated about the probability that a given bid would win the auction. In the MT treatment, participants were required to write the probability that a person would win a monetary auction if she submitted the following bids: 50, 100, 150, 200, 250, 300, 350, 400, 450, 500, 550, 600, 650, $700,750,800,850,900$, and 950 ECU. They had to express their answer as a percentage between $0 \%$ and $100 \%$, inclusive. The estimates were incentivized using a quadratic scoring rule (Brier

\footnotetext{
${ }^{7}$ The valuations were drawn from the interval from 0 to 30 , in order to ensure that the maximum potential gain, when a subject has a valuation of 30 and makes a bid of 0 and wins, would not result in a negative length of time that a subject would be required to spend in the laboratory.
} 
1950; Nyarko and Schotter,2002; Rey-Biel, 2009) that is incentive compatible ${ }^{8}$. The payoff function was the following:

$$
\Pi=\max \left\{0,10-45 *\left(\mathrm{P}_{\mathrm{t}}-\mathrm{P}_{\mathrm{a}}\right)^{2}\right\}
$$

where $\mathrm{P}_{t}$ stands for the indicated probability of winning the auction and $\mathrm{P}_{\mathrm{a}}$ represents the actual probability. The maximum of the two terms in the expression is taken in order to ensure nonnegative payoffs. The subject could win a maximum amount of $10 €$ and a minimum of $0 €$. The actual probabilities, to which the guesses were compared, were estimated by a probit regression conducted on the data from the first session. Subjects were not informed about their performance on this task until the experiment ended. One randomly chosen decision in this task counted toward a participant's earnings.

\subsection{Cognitive Reflection Test}

At the beginning of each session, we also evaluated subjects' willingness/ability to reflect on a decision problem. In both MT and T, we administered the Cognitive Reflection Test (CRT) introduced by Frederick (2005). Each question in the test has an intuitive, though erroneous, answer. However, if the respondent reflects on the problem, the solution is straightforward. This test is widely used in experimental economics (Branas-Garza et al., 2016).

Individuals who register to participate in experiments are likely to have taken part in more than one experiment, and thus may have been exposed to the CRT previously. In order to account for the possibility, the standard questions were modified while preserving their underlying logic ${ }^{9}$. Specifically, we asked the following three questions:

- A shirt and a hat cost $5.50 €$ in total. The shirt costs $5 €$ more than the hat. How much does the hat cost? (correct answer: 25 cents; intuitive erroneous answer: 50 cents)

\footnotetext{
8 The quadratic scoring rule (QSR) assumes that respondents are risk neutral (Starmer, 2000). Adjustments to QSR procedures which account for risk aversion have been proposed by Offerman et al. (2009) and Anderson et al. (2010). These methods have been shown to reduce bias, but not to significantly increase accuracy (Trautman and Kuilen, 2011).

${ }^{9}$ Each individual was asked if they have encountered any of the CRT questions previously. $46 \%$ of the participants reported that they have seen similar questions before. Nevertheless, $23 \%$ of the subjects who answered 0 or 1 question out of three correctly reported that they had already seen the test before. Brañas-Garza et al (2015), in a meta-study, find no significant difference in performance on the CRT test between respondents who have seen the test before and those who have not.
} 
- If it takes 3 people 3 hours to assemble 3 bicycles, how long would it take 90 people to assemble 90 bicycles? (correct answer: 3 hours; intuitive erroneous answer: 90 hours)

- The forest of North Carolina is home to a colony of wild rabbits. Every year, the colony doubles in size. If it takes 24 years for the colony to reach 100.000 rabbits, how long would it take for the colony to reach half of that number? (correct answer: 23 years; intuitive erroneous answer: 12 years)

\subsection{Sequence of activity in each session}

Figure 1 illustrates the timing of events in each of the two treatments. The MT protocol consisted of 4 parts, and the timing is illustrated in the upper half of Figure 1. In the first part, subjects were asked to answer the questionnaire for the CRT test. Participants received $1 €$ for each correct response. In part two, after we carefully explained the design of a monetary all-pay auction and provided some examples, the probability calibration measurement protocol was implemented. It was emphasized that the predictions would not influence payoffs within the actual auctions played (and vice-versa). One prediction, which would be drawn randomly at the end of the experiment, would determine the participant's payoff in part two. In part three, there were sixteen periods of monetary auctions: one period of practice that did not count toward participants' earnings, followed by 15 consecutive auctions that did count. A stranger matching procedure was implemented, so that each subject was grouped randomly with a new group of four other bidders in each period. Finally, one time auction was conducted, and constituted part four of the session in the MT protocol.

The $\mathrm{T}$ protocol consisted of three parts. The sequence of events is shown in the lower panel of Figure 1. The show-up fee was rescaled to 5 Euros to account for the relatively short length of the experiment. The session began by asking the participants to fill in the questionnaire for the CRT test. In part 2, the instructions for the time auction were read. The probability calibration task was then administered, with the elicitation of probabilities in terms of time rather than money. Participants were required to write down the probability that they believed that a person would win the auction if she submitted the following bids: 2, 4, 6, 8, 10, 12, 14, 16, 18, 20, 22, 24, 26 and 28 ECU. The answer had to be expressed as a percentage between $0 \%$ and $100 \%$. Part 3 of the session consisted of one all-pay auction in which the medium of reward was time. 
The next three sections are organized in the following manner. In section four, we consider the data from the all-pay auctions played using money as a medium of reward. In subsection 4.1, we report the aggregate patterns in the data. In subsection 4.2, we consider individual bidding strategies and how they correlate with our measures of sophistication. In subsection 4.3, we analyze the correlates of effective bidding. In section 5, we turn our attention to the time auctions. As for the monetary auctions, the first subsection, 5.1, reviews the overall patterns in the data. 5.2 investigates correlations between of bidding behavior and the sophistication measures, and 5.3, the determinants of effective bidding. In section 6, we provide a comparison between the monetary and time auctions.

\section{Results for the Monetary All-pay Auction}

\subsection{Overall bidding behavior and earnings}

Figure 2 shows the average difference between the observed bids and the Bayesian equilibrium bid levels, pooled across participants and sessions, for each period separately. In the figure, the horizontal axis measures time, the period $(1-15)$ in which the auction occurs. The vertical axis indicates the average difference between the bids submitted in the period and the Risk Neutral Bayesian Equilibrium (RNBE) bid, pooled over all of the MT sessions. Values greater than zero indicate that the average bid was greater than the equilibrium level. The figure reveals that on average, overbidding occurs in every period. The extent of overbidding does decrease over time but remains present, even in the last period.

The MT Protocol

\begin{tabular}{|c|c|c|c|}
\hline $\begin{array}{c}\text { Part 1: CRT } \\
\text { Questionnaire }\end{array}$ & Part 2: Eliciting Beliefs & $\begin{array}{c}\text { Part 3: Monetary All-Pay } \\
\text { Auctions }\end{array}$ & $\begin{array}{c}\text { Part 4: Time All-Pay } \\
\text { Auctions }\end{array}$ \\
\hline
\end{tabular}

The T Protocol

\begin{tabular}{|l|l|l|}
\hline Part 1: CRT Questionnaire & Part 2: Eliciting Beliefs & Part 3: Time All-Pay Auctions \\
\hline
\end{tabular}

Figure 1. Timing of activity in the two treatments 


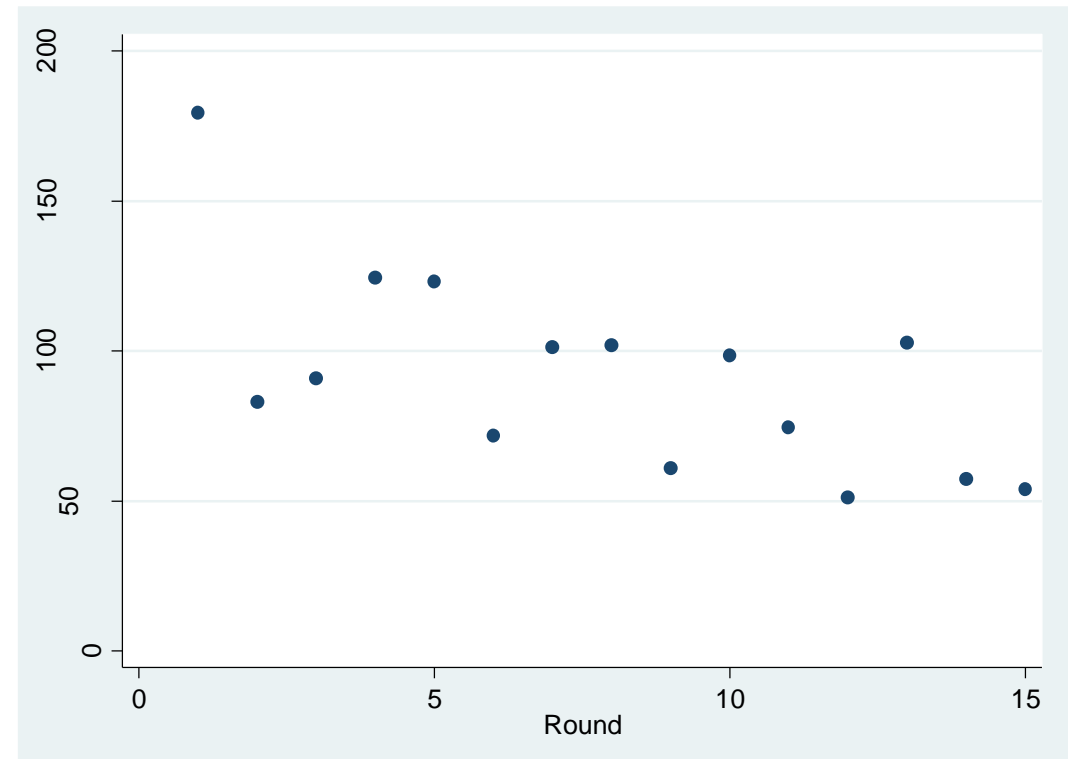

Figure 2. The average difference between the observed bids and RNBE, by period

One consequence of this overbidding is that participants' earnings are lower than predicted. Summary statistics of bidders' earnings are presented in table 1, where aggregate averages across all periods, within each session, as well as the equilibrium outcomes, are recorded. The data show that the average participant lost money during the monetary auctions. Only 4 out of 60 participants made a profit over the 15 auctions, and only 28 failed to lose their entire initial endowment of 1000 ECU. The rest had their losses deducted from their earnings accumulated in other tasks and their show up fee.

The table also displays the average allocative efficiency observed in the auctions, as well as the percentage of auctions in which an efficient allocation occurs, by session. The average efficiency is $82 \%$, and the percentage of auctions which are efficient is $46 \%$. This incidence of efficiency is lower than that typically observed in winner-pay first price auctions ${ }^{10}$.

\footnotetext{
${ }^{10}$ See for example Cox et al. (1988) who report an efficiency of approximately $98 \%$ in first-price winner-pay sealed bid auctions.
} 
Table 1. Average earnings and efficiency in the monetary auction

\begin{tabular}{lcccccc}
\hline & Session 1 & Session 2 & Session 3 & Session 4 & Session 5 & Session 6 \\
\cline { 1 - 5 } All 15 periods & & & & & & \\
Av. Observed Earnings & -100.20 & -61.84 & -103.56 & -47.41 & -115.16 & -130.16 \\
Av. RBNE Earnings & 36.90 & 28.01 & 24.28 & 33.40 & 41.81 & 39.76 \\
Efficiency & $87 \%$ & $71 \%$ & $85 \%$ & $73 \%$ & $89 \%$ & $86 \%$ \\
\% of Auctions that are & $63 \%$ & $30 \%$ & $40 \%$ & $37 \%$ & $60 \%$ & $47 \%$ \\
Efficient & & & & & & \\
\hline
\end{tabular}

Note: The third row of the table, labeled Efficiency indicates the winner's valuation, divided by the highest valuation any player has in the period, averaged across all 15 periods, for each session. The last row is the percentage of instances in which the bidder with the highest valuation is awarded the item.

\subsection{Bidding behavior at individual level}

To study the reasons for the lower than equilibrium level of earnings, we analyze the relationship between valuations and bids at the individual level. Figure 3 displays all bids placed in the last five auction periods. In the figure, the horizontal axis denotes an individual's valuation and the vertical axis is the bid that the individual submits. The bids in each round are shown in a different color and the Bayesian equilibrium is depicted by a smooth line.

Heterogeneous behavior is observed among the participants, but there seem to be a few general bidding rules that many subjects use. The incidence of the three most common rules is given in Table 2. The first is a rule of bidding zero, regardless of one's valuation. Strictly speaking, zero bidding is a requirement that $b_{i}\left(v_{i}\right)=0$, for all $v_{i}$. However, in our classification, we admit any strategy $b_{i}\left(v_{i}\right) \leq 20$ for all $v_{i}$ into the category. We consider a bidder to employ a zero bidding strategy is she uses the strategy in each of the last five auction periods. Bidding zero is quite common, and it is the strategy employed by $25 \%$ of bidders. Zero bidding is less common for high valuations than for low ones. Zero bidding represents a strategy that almost always leads to a zero payoff. $^{11}$

\footnotetext{
${ }^{11}$ There is a very small probability of a strictly positive payoff if all bidders bid 0 , and the tie is resolved in one's favor. There is no possibility of losing money when one bids zero.
} 


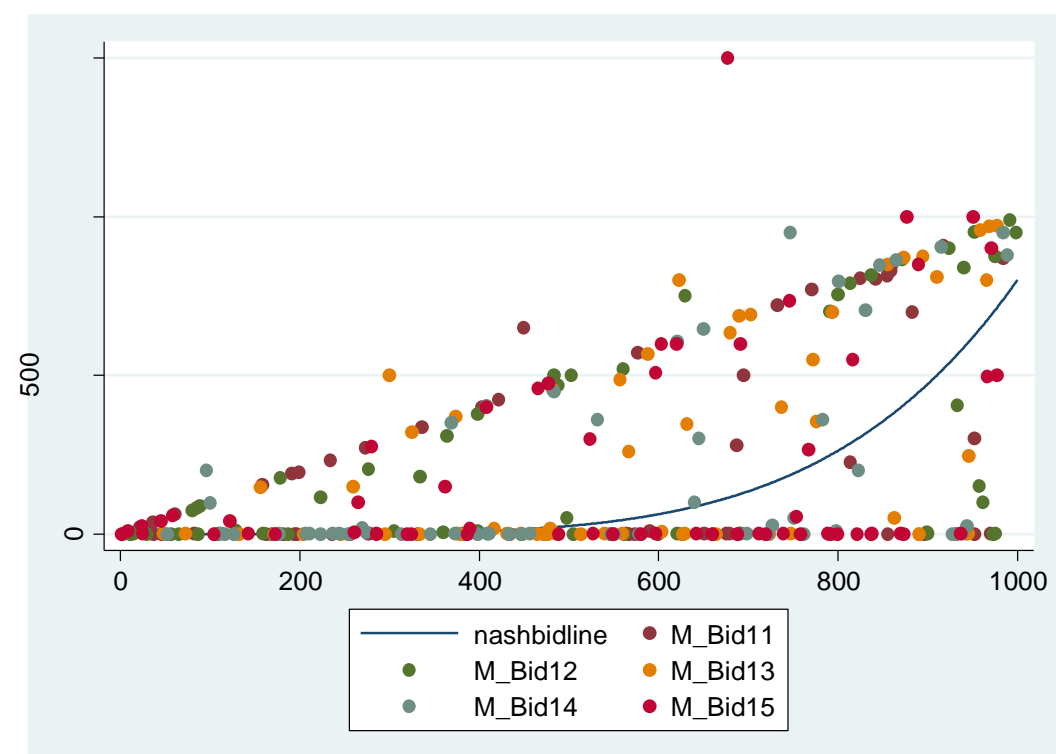

Figure 3. The Relationship between bids and valuations, Last 5 periods

The figure also shows that submitting a bid equal to one's valuation is also common. We call this diagonal bidding and it is defined as $b_{i}\left(v_{i}\right)=v_{i}$, though for purpose of classification we consider $b_{i}\left(v_{i}\right) \varepsilon\left[v_{i}-50, v_{i}+50\right]$ to fall into the category. $13.3 \%$ of players are classified as diagonal bidders, meaning that they employ a diagonal strategy in the last five periods. Diagonal bidding is a poor strategy that is guaranteed to yield a payoff of either zero, in the event one's bid is the highest in the group, or negative if it is not the highest. The diagonal bidding phenomenon is common both for low and high valuations.

A third type of bidding behavior that is not as easy to observe in Figure 3, but that is common, is threshold bidding. A threshold bidder bids zero if her valuation is below a threshold, and then bids a relatively high amount close to her valuation, when the valuation exceeds the threshold. In other words, a threshold bidder pursues a strategy of $b_{i}\left(v_{i}\right)=0$, for all $v_{i} \leq \mathrm{v}^{*}$, and $b_{i}\left(v_{i}\right)=v_{i}$ for all $v_{i}>\mathrm{v}^{*}$, for some $\mathrm{v}^{*}$. We will say that individual $i$ 's behavior meets the criterion to be considered in this category if there exists some $\mathrm{v}^{*}$, such that $b_{i}\left(v_{i}\right) \leq 20$ for all $v_{i} \leq \mathrm{v}^{*}$, and $b_{i}\left(v_{i}\right) \varepsilon\left[v_{i}-50, v_{i}\right.$ $+50]$ for all $v_{i}>\mathrm{v}^{*}$. Threshold bidding has been noted in other studies of contests by Noussair and Silver (2006) and Mueller and Schotter (2010). Table 2 shows that a full 33.3\% of bidders employ this strategy in the last 5 periods. 
We now consider which factors correlate with the use of each of these three strategies. Table 2 shows the percentage of bidders classified as types corresponding to the commonly used strategies described above. The table shows that females are more likely than males to use a diagonal bidding strategy and males are relatively more likely to be threshold bidders. Zero or threshold bidders are more likely to have high CRT scores and to be well-calibrated than diagonal bidders are. Diagonal bidders also earn less and deviate more from equilibrium than the other two types.

Table 2. Bidding strategies, gender, cognitive ability and earnings

\begin{tabular}{|c|c|c|c|c|}
\hline & Zero bidders & Diagonal bidders & Threshold bidders & Other \\
\hline $\begin{array}{c}\text { Percentage using } \\
\text { strategy overall }\end{array}$ & $25 \%$ & $13.33 \%$ & $33.33 \%$ & $28.33 \%$ \\
\hline $\begin{array}{c}\text { Percentage using } \\
\text { strategy who are } \\
\text { male* }\end{array}$ & $46 \%$ & $25 \%$ & $55 \%$ & $47 \%$ \\
\hline $\begin{array}{c}\text { Percentage using } \\
\text { strategy who have } \\
\text { CRT=2,3 }\end{array}$ & $86 \%$ & $38 \%$ & $85 \%$ & $53 \%$ \\
\hline $\begin{array}{c}\text { Percentage using } \\
\text { strategy who are } \\
\text { well-calibrated }\end{array}$ & $36 \%$ & $50 \%$ & $36 \%$ & 1.47 \\
\hline $\begin{array}{c}\text { Average CRT } \\
\text { Average Earnings } \\
\text { per round }\end{array}$ & -41.91 & -152.34 & -93.54 & -109.70 \\
\hline $\begin{array}{c}\text { Average Abs } \\
\text { deviation from } \\
\text { RNBE }\end{array}$ & 167.49 & 332.39 & 157.90 & 257.02 \\
\hline
\end{tabular}

*The gender information was not collected for 2 subjects

\subsection{Correlates of effective bidding}

We use two different notions of effectiveness of bidding strategies. The first is to say that an effective strategy is one that is close to the equilibrium level. The second criterion is to define an effective strategy as one that yields high earnings. Table 3 below shows which factors correlate with both criteria: with Absolute Deviation of bid from equilibrium, and with earnings. The data

\footnotetext{
${ }^{12}$ We consider subjects to be well-calibrated if their accuracy in the probability calibration task was higher than the average accuracy level. In our data $39.6 \%$ of the subjects ( 23 out of 58 ) are well calibrated. Therefore, the distribution of performance in the task was right-skewed.
} 
in Table 3 show that better probability calibration is correlated with bidding closer to equilibrium, and with higher earnings. Overestimating the probability of winning in our calibration task is correlated with overbidding and with lower earnings. This pattern is consistent with those players with pessimistic beliefs giving up on trying to win the auction, and as a consequence achieving higher earnings. Males in our sample bid less than females, exhibit less miscalibration in our task, and earn more on average in the monetary auction. Individuals with higher CRT scores also bid lower and earn more.

Table 3. Cross tabulation of correlations of subject characteristics and bidding outcomes in the monetary auction

\begin{tabular}{|c|c|c|c|c|c|c|}
\hline Correlation & $\begin{array}{c}\text { Absolute } \\
\text { Bid } \\
\text { Deviation }\end{array}$ & $\begin{array}{c}\text { Avg. } \\
\text { Earnings }\end{array}$ & $\begin{array}{c}\text { Absolute } \\
\text { probability } \\
\text { miscalibration }\end{array}$ & $\begin{array}{c}\text { Probability } \\
\text { Miscalibration }\end{array}$ & Male & $\begin{array}{c}\text { High } \\
\text { ability } \\
(\mathrm{CRT}=2 \mid 3) \\
\end{array}$ \\
\hline $\begin{array}{c}\text { Absolute Bid } \\
\text { Deviation }\end{array}$ & 1 & & & & & \\
\hline Avg. Earnings & $-0.63 * * *$ & 1 & & & & \\
\hline $\begin{array}{c}\text { Absolute } \\
\text { Probability } \\
\text { Miscalibration }\end{array}$ & $0.31 * *$ & $-0.41 *$ & 1 & & & \\
\hline $\begin{array}{c}\text { Probability } \\
\text { Miscalibration }\end{array}$ & $0.35 * * *$ & $-0.32 * *$ & $0.43 * * *$ & 1 & & \\
\hline Male & $-0.23^{*}$ & $0.37 * *$ & $-0.30 * *$ & $-0.32 * *$ & 1 & \\
\hline $\begin{array}{l}\text { High ability } \\
(\mathrm{CRT}=2 \mid 3)\end{array}$ & $-0.30 * *$ & 0.16 & $-0.28 *$ & $-0.21 *$ & 0.20 & 1 \\
\hline
\end{tabular}

Note: Absolute Bid Deviation is calculated as $a v g|b-\tilde{b}|$, where $b$ is the actual bid and $\tilde{b}$ is the Bayesian equilibrium bid. Probability miscalibration is calculated as $\operatorname{avg}\left(\frac{\gamma-\tilde{\gamma}}{\tilde{\gamma}}\right)$, where $\gamma$ is the actual probability and $\tilde{\gamma}$ is the submitted probability. This measure captures whether there is underestimation or overestimation; Absolute probability miscalibration is calculated as avg $\left|\frac{\gamma-\widetilde{\gamma}}{\widetilde{\gamma}}\right|$ and it captures the magnitude of the miscalibration.

Since CRT scores correlate negatively with deviations from the Bayesian equilibirum, we compare behavior between individuals with low and high CRT scores in more detail. The differences in bidding behavior between the two groups is illustrated in Figure 4. In the figure, the horizontal axes are individuals' valuations and the vertical axes indicate the corresponding bids. Figure 4a shows the behavior of individuals who scored either 0 or 1 on the CRT test, while Figure $4 \mathrm{~b}$ illustrates the data for those who answered at least two of the three questions correctly. It can be observed that diagonal bidding is largely confined to those with lower CRT scores. 


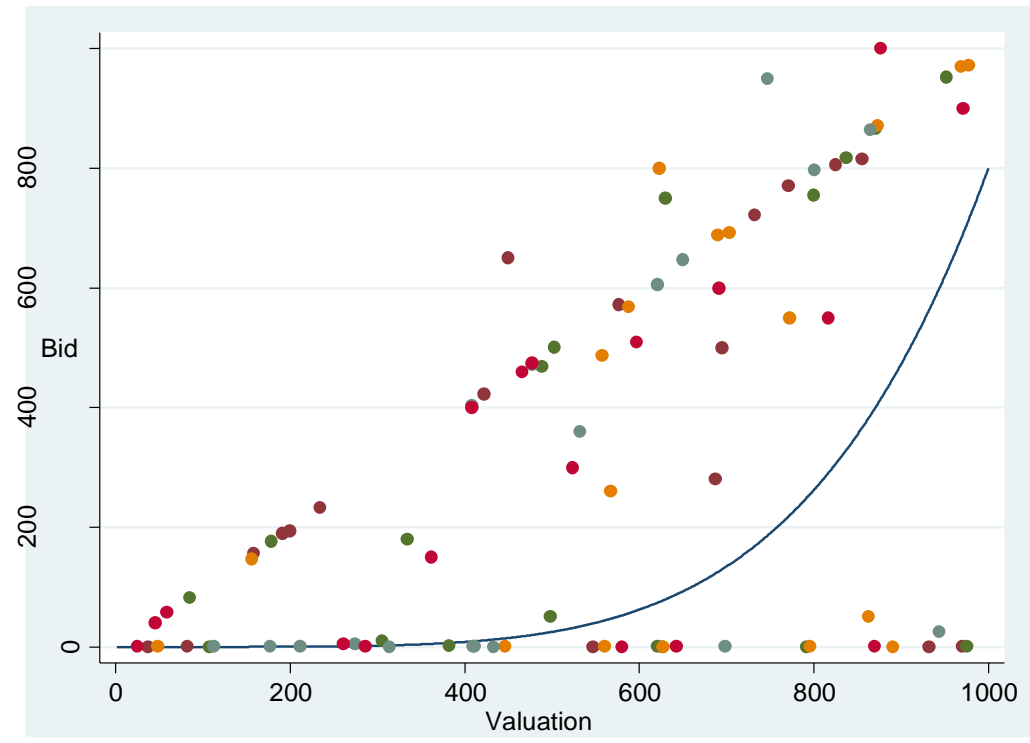

Figure 4a. Bids and valuations, last 5 periods, CRT $<2$ (low scores), equilibrium also shown

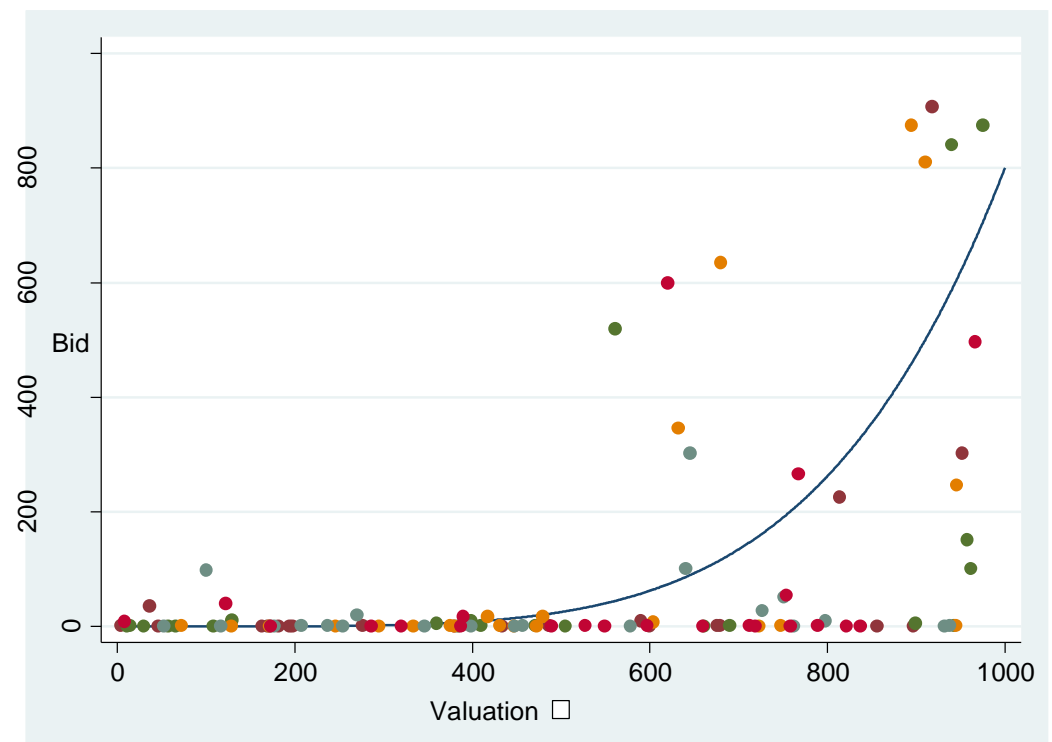

Figure $4 \mathrm{~b}$. Bids and valuations, last 5 periods, $C R T \geq 2$ (high scores), equilibrium also shown

To further analyze whether individual probability calibration and cognitive abilities correlate with bids and earnings, when controlling for other variables, we conduct robust OLS regressions, using cognitive ability and probability calibration as independent variables and the 
average absolute deviation from the RNBE or average earnings as the dependent variable ${ }^{13}$. The estimation results are reported in Table 4. They show that individuals' CRT scores are correlated with bidding closer to the equilibrium level and with earnings. They also reveal that those who are better calibrated earn more money, even when controlling for CRT. Males earn more than females, controlling for calibration and CRT scores. Miscalibration is associated with bidding farther way from equilibrium when CRT is not controlled for. When CRT is controlled for, miscalibration is associated with higher bidding. Thus, CRT is correlated with lower bids, while miscalibration is correlated with deviations from equilibrium. This results in a positive relationship between CRT and earnings, since low CRT people overbid relative to the equilibrium. It also results in lower earnings for miscalibrated people, who are more likely to deviate from equilibrium, in particular by overbidding.

Table 4. Determinants of overbidding, differences from equilibrium, and earnings (a) Absolute difference between bid and RBNE

\begin{tabular}{ccccc}
\hline & $(\mathbf{1})$ & $\mathbf{( 2 )}$ & $\mathbf{( 3 )}$ & $\mathbf{( 4 )}$ \\
\hline & Abs. Average & Abs. Average & Abs. Average & Abs. Average \\
Bid-RNBE & Bid-RNBE & Bid-RNBE & Bid-RNBE \\
CRT & -35.69 & -34.02 & & -39.31 \\
& $(-3.42)^{* * *}$ & $(-3.17)^{* * *}$ & & $(-3.90)^{* * *}$ \\
Abs. probability & 52.58 & 50.15 & 75.037 & \\
miscalibration & $(1.39)$ & $(1.36)$ & $(2.25)^{* *}$ & \\
Male & & -14.90 & -29.13 & -22.15 \\
& & $(-0.67)$ & $(-1.16)$ & $(-0.94)$ \\
Constant & 259.62 & 265.59 & 196.86 & 298.20 \\
& $(8.09)^{* * *}$ & $(8.37)^{* * *}$ & $(8.58)^{* * *}$ & $(12.62)^{* * *}$ \\
& & & & 58 \\
Observations & 58 & 56 & 56 & $23 \%$ \\
R-squared & $24 \%$ & $25 \%$ & $12 \%$ & 58 \\
\hline
\end{tabular}

Note: The dependent variable is the absolute difference between the observed bid and Bayesian equilibrium bid, averaged over the 15 monetary auction periods. Each individual participant is a unit of observation. Abs. probability miscalibration represents the absolute difference between the probability of winning with a certain bid as indicated by a participant and the actual probability, averaged over all 19 calibration questions. OLS regression, t-value based on robust standard errors in parenthesis; ${ }^{* * *} / * * *$ indicates statistical significance at $10 \%, 5 \%$ and $1 \%$ level, repectively.

\footnotetext{
${ }^{13}$ There are two individuals who believed that there was a $0 \%$ chance of winning the auction for every bid between 50 and 950 ECU. They were excluded from the analysis.
} 
(b) Average earnings

\begin{tabular}{ccccc}
\hline & $\mathbf{( 1 )}$ & $\mathbf{( 2 )}$ & $\mathbf{( 3 )}$ & $\mathbf{( 4 )}$ \\
\hline & Average & Average & Average & Average \\
& Earnings & Earnings & Earnings & Earnings \\
CRT & 19.18 & 15.50 & & 22.56 \\
& $(2.45)^{* *}$ & $(1.90)$ & & $(2.58)^{* *}$ \\
Avg. Abs probability & -83.50 & -73.11 & -84.45 & \\
miscalibration & $(-2.85)^{* * *}$ & $(-2.69)^{* * *}$ & $(-3.02)^{* * *}$ & \\
Male & & 39.18 & 45.68 & 44.51 \\
& & $(1.88)$ & $(2.25)^{* *}$ & $(2.07)^{*}$ \\
Constant & -92.49 & -107.42 & -76.10 & -155.84 \\
& $(-3.94)^{* * *}$ & $(-4.90)^{* * *}$ & $(-3.55)^{* * *}$ & $(-7.34)^{* * *}$ \\
Observations & 58 & 56 & 56 & 58 \\
R-squared & $21 \%$ & $27 \%$ & $26 \%$ & $19 \%$ \\
\hline
\end{tabular}

(c) Average difference between bid and RNBE

\begin{tabular}{ccccc}
\hline & $\mathbf{( 1 )}$ & $\mathbf{( 2 )}$ & $\mathbf{( 3 )}$ & $\mathbf{( 4 )}$ \\
\hline & Average Bid- & Average Bid- & Average Bid-RNBE & Average Bid- \\
& RNBE & RNBE & & RNBE \\
CRT & -33.05 & -31.99 & & -39.31 \\
& $(-3.18)^{* * *}$ & $(-2.96)^{* * *}$ & & $(-3.90)^{* * *}$ \\
Probability miscalibration & 53.19 & 52.60 & 74.20 & \\
& $(2.71)^{* * *}$ & $(2.52)^{* *}$ & $(4.33)^{* * *}$ & \\
Male & & -10.86 & -21.06 & -22.15 \\
& & $(-0.48)$ & $(-0.88)$ & $(-0.94)$ \\
Constant & 268.36 & 270.94 & 211.45 & 298.20 \\
& $(11.14)^{* * *}$ & $(10.62)^{* * *}$ & $(13.38)^{* * *}$ & $(12.62)^{* * *}$ \\
Observations & 60 & 58 & 58 & 58 \\
R-squared & $29 \%$ & $29 \%$ & $18 \%$ & $23 \%$ \\
\hline
\end{tabular}

Our findings regarding behavior and outcomes in the monetary auctions are summarized as result 1 .

Result 1: In the auctions where money is the medium of reward, bidding exceeds the riskneutral Bayesian equilibrium levels on average. There is overdissipation of the available rent, 
and average earnings of bidders are negative. Those players who are more sophisticated, as measured by both CRT scores and probability calibration, bid lower and receive greater earnings.

\section{Results for the time all-pay auction}

\subsection{Time auction statistics and individual behavior}

We start by considering the bidding strategies employed in the time auctions. Figure 5 plots the relationship between valuation and bid for the time auction played under both protocols. The overall pattern in bidding behavior does not seem to be very dissimilar, though under $\mathrm{T}$ it seems that overbidding is more widespread for high valuations than under MT.
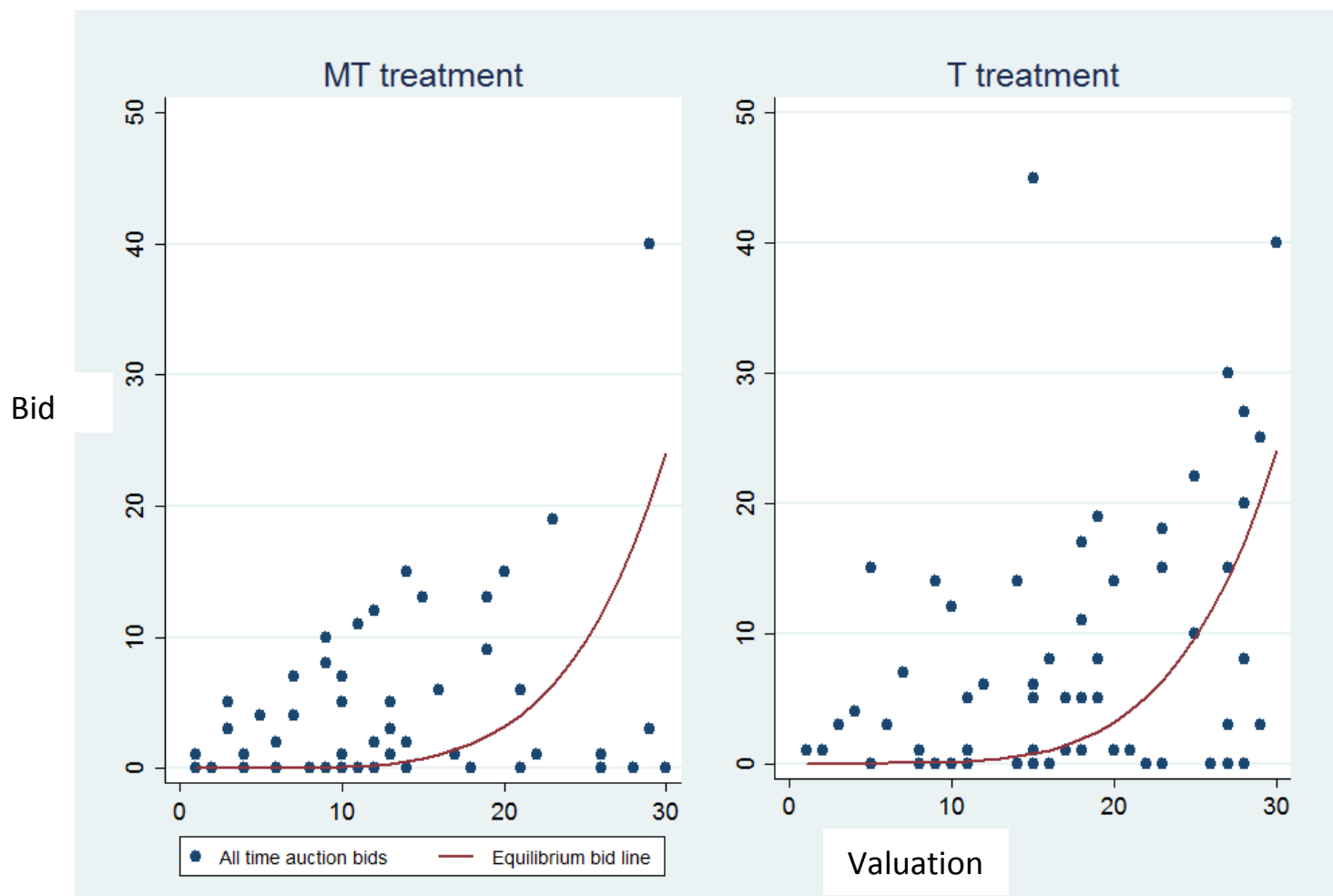

Figure 5. Bidding behavior in all time auctions in the MT (left panel) and T (right panel) protocols

Table 5 provides some comparison statistics for the time auction in both $\mathrm{T}$ and MT. The table shows that the extent of overbidding, in terms of the average percentage overbid compared 
to the equilibrium level, is comparable under the two protocols. Average bidder earnings are negative in the time auctions, as in the monetary auctions. The efficiency percentage of auctions is lower in the time than in the monetary auctions, indicating that there is more heterogeneity in bidding strategies in the time auctions.

Table 5. Summary Statistics for the time auction in both T and MT

\begin{tabular}{lrr}
\hline & $\begin{array}{r}\text { Time all-pay auction } \\
\text { ( T treatment ) }\end{array}$ & $\begin{array}{r}\text { Time all-pay auction } \\
\text { (MT treatment) }\end{array}$ \\
\cline { 2 - 3 } & Mean & Mean \\
Bid-RNBE (in minutes) & 3.26 & 1.39 \\
Abs |Bid-RNBE| (in & 6.66 & 5.32 \\
minutes) & & \\
AV. (Bid-RNBE) $>\mathbf{0}$ & 7.83 & 6.38 \\
Av. Minutes Waited & 33.17 & 31.05 \\
Earnings (in minutes) & -3.17 & -1.05 \\
Efficiency & $79 \%$ & $72 \%$ \\
\hline
\end{tabular}

Note: Bid-RNBE is the difference between the actual bid and the risk neutral Bayesian equilibrium bid. Abs (Bid$\mathrm{RNBE}$ ) is the absolute difference between the actual bid and the risk neutral Bayesian equilibrium bid Av(Bid-RNBE) $>0$ indicates the average overbid.

Av. earnings are net of initial waiting time, so that for example, an individual who is required to wait 32 minutes has earnings of -2 minutes.

Efficiency is the valuation (time exemption) of the winning bidder divided by highest valuation among bidders.

Table 6 documents some correlates of bidding behavior and earnings in the time auction. The table reveals that those who bid higher receive lower earnings. Unlike in the monetary auctions, probability miscalibration is not correlated with bidding behavior or earnings. There is a strong gender effect. Women bid on average 5.78 minutes which is significantly more than their male counterparts, who bid only 0.78 minutes $\left(\mathrm{p}=0.015, \mathrm{~N}_{1}=29, \mathrm{~N}_{2}=31\right.$, $\mathrm{t}$-test). This is consistent with some of the literature on monetary auctions (Chen et al., 2013) that finds that women bid higher than men. However, average earnings remain similar for both genders. 
Table 6a: Correlations between key variables in the time auction under the T protocol

\begin{tabular}{|c|c|c|c|c|c|c|c|}
\hline Correlation & $\begin{array}{c}\text { Absolute } \\
\text { Av. Bid }\end{array}$ & Av. Bid & $\begin{array}{c}\text { Av. } \\
\text { Earnings }\end{array}$ & $\begin{array}{c}\text { Absolute } \\
\text { probability } \\
\text { miscalibration }\end{array}$ & $\begin{array}{c}\text { Probability } \\
\text { Miscalibration }\end{array}$ & Male & $\begin{array}{c}\text { High } \\
\text { ability } \\
\text { (CRT=2|3) }\end{array}$ \\
\hline $\begin{array}{c}\text { Absolute Av. } \\
\text { Bid }\end{array}$ & 1 & & & & & & \\
\hline Av. Bid & $0.53^{* * *}$ & 1 & & & & & \\
\hline Av. Earnings & $-0.49 * * *$ & $-0.58^{* * *}$ & 1 & & & & \\
\hline $\begin{array}{c}\text { Absolute } \\
\text { probability } \\
\text { miscalibration }\end{array}$ & -0.067 & -0.070 & -0.10 & 1 & & & \\
\hline $\begin{array}{c}\text { Probability } \\
\text { Miscalibration }\end{array}$ & 0.09 & 0.15 & -0.02 & -0.12 & 1 & & \\
\hline Male & -0.20 & $-0.28 * *$ & 0.12 & 0.08 & $-0.23^{*}$ & 1 & \\
\hline $\begin{array}{c}\text { High ability } \\
\text { (CRT=2|3) }\end{array}$ & -0.001 & 0.02 & -0.068 & -0.11 & -0.12 & 0.10 & 1 \\
\hline
\end{tabular}

Table 6b: Correlations between key variables in the time auction played under the MT protocol ${ }^{14}$

\begin{tabular}{|c|c|c|c|c|c|}
\hline Correlation & $\begin{array}{c}\text { Absolute } \\
\text { Av. Bid }\end{array}$ & Av. Bid & $\begin{array}{c}\text { Av. } \\
\text { Earnings }\end{array}$ & Male & $\begin{array}{c}\text { High } \\
\text { ability } \\
\text { (CRT=2|3) }\end{array}$ \\
\hline $\begin{array}{c}\text { Absolute Av. } \\
\text { Bid }\end{array}$ & 1 & & & & \\
\hline Av. Bid & -0.092 & 1 & & & \\
\hline Av. Earnings & -0.24 & -0.23 & 1 & & \\
\hline Male & -0.18 & $-0.40^{* * *}$ & 0.16 & 1 & \\
\hline $\begin{array}{c}\text { High ability } \\
\text { (CRT=2|3) }\end{array}$ & $-0.34 * * *$ & $-0.25 *$ & 0.05 & 0.14 & 1 \\
\hline
\end{tabular}

\subsection{Correlates of Effective Strategies}

We now consider whether some player characteristics are predictive of bidding closer to equilibrium or for earning greater payoffs. The results for the T protocol are shown in Table 7. The analogous estimates for MT are similar and are given in Appendix C. The regressions presented in the table indicate that CRT score and calibration are not correlates of bids or earnings in the time auction. The R-squared values show that the variables considered in the regressions explain much less variation than in the money auctions. The only significant variable is the tendency for male subjects to bid lower than females. While males bid lower than females, they are no closer to the equilibrium level, and they do not achieve greater earnings than females. This gender difference

\footnotetext{
${ }^{14}$ The probability calibration data are not included in table $6 \mathrm{~b}$, because in the MT protocol, the calibration was conducted for the money auction, and not for the time auction.
} 
might reflect different utility functions for time, ${ }^{15}$ or rather the tendency to the use different bidding strategies, and our experimental design does not allow us to distinguish between these two general explanations.

Table 7. OLS Results - Time auction (treatment T)

(a) Absolute deviation from RNBE bid

\begin{tabular}{ccccc}
\hline & $(1)$ & $(2)$ & $(3)$ & $(4)$ \\
\hline & Abs. Bid-RNBE & Abs. Bid-RNBE & Abs. Bid-RNBE & Abs. Bid-RNBE \\
CRT & -0.94 & -0.85 & & -0.66 \\
& $(-1.32)$ & $(-1.11)$ & & $(-3.90)$ \\
Abs. avg. probability & -2.95 & -2.44 & -1.34 & \\
calibration & & & & \\
& $(-0.93)$ & $(-0.74)$ & $(-0.49)$ & -2.83 \\
Male & & -2.70 & -2.85 & $(-1.45)$ \\
& & $(-1.33)$ & $(-1.43)$ & 9.26 \\
Constant & 9.51 & 10.52 & 8.70 & $(5.06)^{* * *}$ \\
& $(4.45)^{* * *}$ & $(4.63)^{* * *}$ & $(5.22)^{* * *}$ & 60 \\
Observations & 60 & 60 & 60 & $5 \%$ \\
R-squared & $2.5 \%$ & $5.8 \%$ & $4 \%$ & \\
\hline
\end{tabular}

Note: dependent variable: Absolute difference between the risk neutral Bayesian Nash equilibrium and observed bid in the time auction. Each individual participant is the unit of observation. Abs. average probability calibration is the absolute difference between the probability of winning with a certain bid indicated by the participants in the calibration task and the actual probability, averaged across all 19 probability levels. OLS regression, $\mathrm{t}$-value based on robust s.e. in parenthesis; * ${ }^{* *} / * * *$ stands for statistical significance at $10 \%, 5 \%$ and $1 \%$ level, respectively

(b) Earnings

\begin{tabular}{ccccc}
\hline & $\mathbf{( 1 )}$ & $\mathbf{( 2 )}$ & $\mathbf{( 3 )}$ & $\mathbf{( 4 )}$ \\
\hline CRT & Earnings & Earnings & Earnings & Earnings \\
& -0.001 & -0.07 & & 0.15 \\
Abs. Average & $(-0.00)$ & $(-0.09)$ & & $(0.21)$ \\
probability calibration & -2.50 & -2.86 & -2.77 & \\
& & & & \\
Male & $(-0.73)$ & $(-0.81)$ & $(-0.88)$ & 1.73 \\
& & 1.88 & 1.87 & $(0.89)$ \\
Constant & -2.12 & $(0.93)$ & $(0.97)$ & -4.31 \\
& $(-0.86)$ & -2.83 & -2.98 & $(-2.48)^{* *}$ \\
Observations & 60 & $(-1.18)$ & $(-1.75)^{*}$ & 60 \\
Goodness of fit: & $0 \%$ & 56 & 60 & $1 \%$ \\
R-squared & & $2 \%$ & $2 \%$ & \\
\hline
\end{tabular}

${ }^{15}$ There is some evidence that women are more patient than men. See for example Rubalcava et al. (2009). 


\begin{tabular}{ccccc}
\hline & $(1)$ & $(2)$ & $(3)$ & $(4)$ \\
\hline CRT & Bid-RNBE & Bid-RNBE & Bid-RNBE & Bid-RNBE \\
& -0.002 & .058 & & -.002 \\
Avg. probability & $(-0.00)$ & $(0.06)$ & & $(-0.00)$ \\
calibration & 4.02 & 2.40 & 2.39 & \\
& & & & \\
Male & $(1.63)$ & $(1.04)$ & $(1.01)$ & -5.32 \\
& & -4.92 & -4.92 & $(-2.19)^{* *}$ \\
Constant & 3.73 & $(-2.04)^{* *}$ & $(-2.07)^{* *}$ & 6.01 \\
& $(1.64)$ & 5.98 & 6.08 & $(2.31)^{* *}$ \\
Observations & 60 & $(2.27)^{* *}$ & $(3.06)^{* * *}$ & 60 \\
Goodness of fit: & $2 \%$ & 60 & 60 & $8 \%$ \\
R-squared & & $9 \%$ & $9 \%$ & \\
\hline
\end{tabular}

Our findings regarding outcomes in the time auctions are collected as our result 2.

Result 2: In the time auction, bidding exceeds risk-neutral Bayesian equilibrium levels on average. There is overdissipation of the available rent. There is no relationship between sophistication and bidding behavior or earnings. Women bid significantly higher than men.

\section{Comparison between contests for money and time}

In this section we compare bidding behavior between the auctions for time and money ${ }^{16}$. We present the data from both the T and MT treatments. They are treated as distinct comparisons, due to the potential existence of differences between the two treatments. Comparison between the money and the time auctions in MT are conducted within-subject. The money auctions and the time auctions in treatment $\mathrm{T}$ are compared between-subject.

In the first monetary auction, individuals bid above the equilibrium on average by 9.41 units, compared to 7.83 units in the time auction in $\mathrm{T}$ and 6.38 units in the time auction in MT. Thus, the average extent of overbidding is comparable for money and time. We test whether deviations from the equilibrium in the first monetary auction in MT and the time auction in treatment $\mathrm{T}$, are significantly different. This comparison is important because individual have a

\footnotetext{
${ }^{16}$ As noted earlier, observations in the monetary auctions were normalized in order to make the two auctions comparable, by multiplying the deviations from the Bayesian Equilibrium by 3/100.
} 
comparable degree of prior experience in the two situations. We observe no significant difference (Prob $\left.>|\mathrm{z}|=0.17, \mathrm{~N}_{1}=60, \mathrm{~N}_{2}=60, \mathrm{MWU}\right)^{17}$. Overbidding is significantly lower in the time auction played in the MT treatment when compared with the first monetary auction in MT (Prob $>|z|=$ $\left.0.007, \mathrm{~N}_{1}=60, \mathrm{~N}_{2}=60, \mathrm{MWU}\right)$. In order to account for the experience accumulated during the monetary auctions, we also compare the overbidding behavior between the last monetary auction ${ }^{18}$ and the time auction played immediately thereafter. There is no significant difference in average deviation from equilibrium between the two auctions (Prob $>|z|=0.3529, N_{1}=60, N_{2}=60, M W U$ ). This confirms that bidding moves closer to equilibrium levels with experience.

However, efficiency, at $75 \%$, is on average 14 percentage points lower in the time auctions than in the monetary auction. This indicates that there is greater heterogeneity in the bidding strategies employed in the time than in the monetary auction. While money is valued more or less similarly among disparate individuals, time appears to be treated differently by different people.

In terms of earnings, individuals lose on average 2.79 units less (43\% less) in the time auctions compared to the first monetary auction. This may be because of the greater incidence of diagonal bidding in the money than in the time auction. Table 8 shows the prevalence of the types of bidding behavior introduced in section 4, in the different auctions. Zero bidding is more common in the time auction played in the MT treatment, in which bidders have more overall experience coming from the monetary auctions played, by the time they play the time action. Diagonal bidding ${ }^{19}$ is observed in $18 \%$ of possible instances in time all-pay auctions, considerably less frequently than in the monetary auction.

There is some correlation between the strategy a given individual uses in a monetary auction and in the subsequent time auction in MT. A significant correlation between the use of a diagonal bidding strategy in the last five periods of the money auction and in the following time auction exists $(\rho=.45, p<.001)$, though there is no similar correlation for zero bidding $(\rho=.07$, $p>.1)$. Earnings are positively correlated in the two types of auction $(\rho=.26, p=.04)$.

\footnotetext{
17 Using a one-tailed t-test we find significant results at $10 \%$ level (all of the other significance tests in this paper are two tailed) suggesting that subjects tend to overbid in the monetary auction $\left(\operatorname{Pr}(T>t)=0.0971, N_{1}=60, N_{2}=60, \mathrm{t}-\right.$ test).

${ }^{18}$ In order to make the time auction comparable to the monetary, the last monetary auctions are normalized in a similar manner as the first monetary auction, by scaling down the values and bids by a factor of 3/100.

${ }^{19}$ Within the context of the time auction, diagonal bidders are individuals who bid $+/-5 \%$ of their time exemptions and zero bidders are individuals who bid 0 minutes. In the context of the first monetary all-pay auction, the same conditions apply as the ones described in section 4.2.
} 
Table 8. Bidding strategies in the first monetary auction and the time auction

\begin{tabular}{|l|c|c|c|}
\hline & $\begin{array}{c}\text { Monetary all-pay auction } \\
\left(\mathbf{1}^{\text {st }} \text { auction, MT treatment }\right)\end{array}$ & $\begin{array}{c}\text { Time all-pay auction } \\
(\mathbf{T} \text { treatment })\end{array}$ & $\begin{array}{c}\text { Time all-pay auction } \\
\text { (MT treatment) }\end{array}$ \\
\hline Zero bidders & Percentage & Percentage & Percentage \\
\hline Diagonal bidders & $19 / 60(31 \%)$ & $15 / 60(25 \%)$ & $24 / 59(41 \%)$ \\
\hline
\end{tabular}

Figure 6 plots the relationship between the valuation and bid for the time auctions and the first monetary auction in MT. The impression one gains from the figure is that using time as a medium of reward creates more diversity in terms of bidding behavior compared to money.

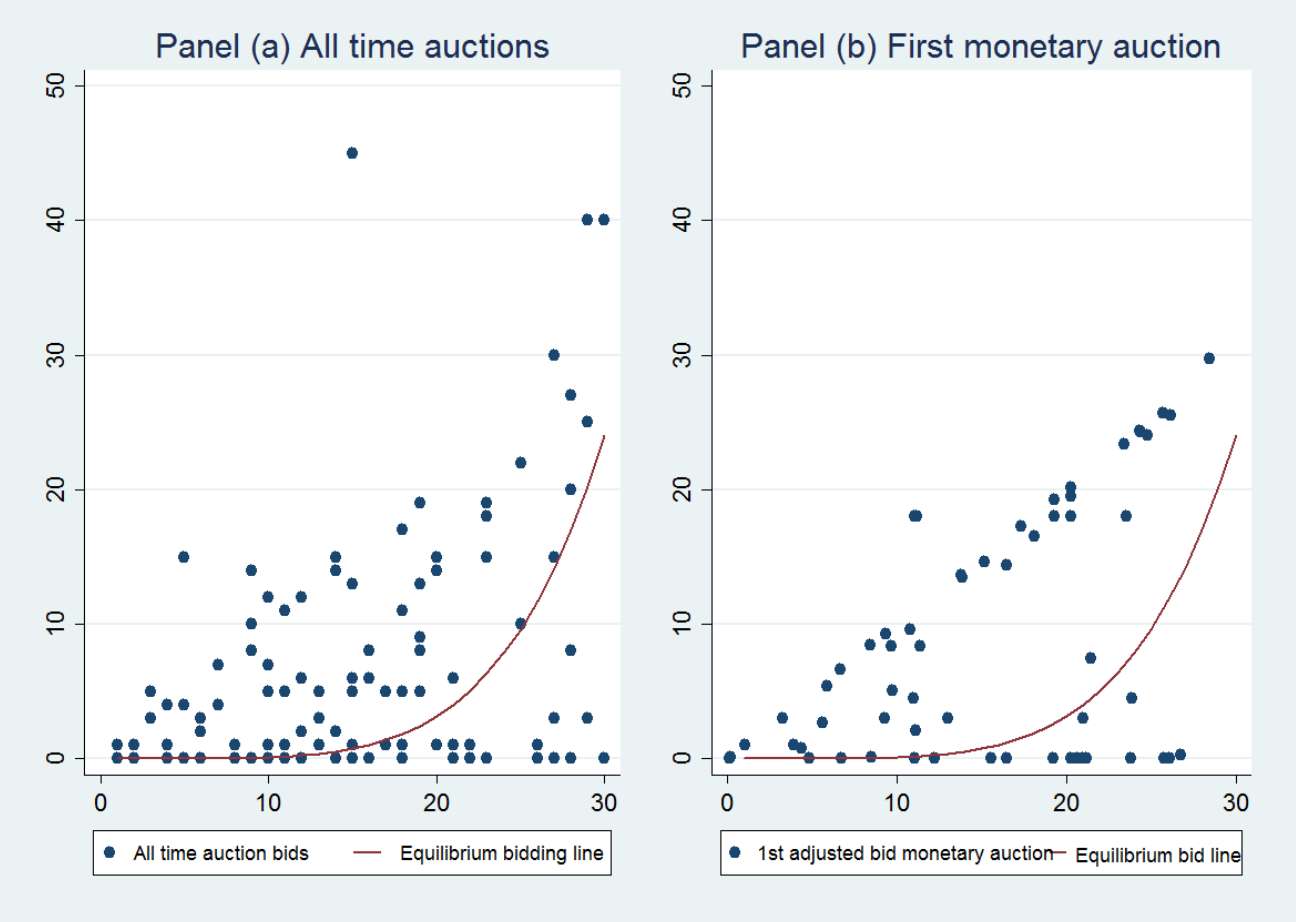

Figure 6. Bidding behavior in all time auctions (panel 1) and in the monetary auction (panel 2)

Figure 7 illustrates the relationship between bidding behavior and CRT score. The panel on the left contains the data from the first monetary auction while that on the right corresponds to the time auction played in the $\mathrm{T}$ protocol. Within each panel, the behavior of individuals with relatively low CRT scores is depicted on the left and that of those with high CRT scores is shown 
on the right. The plots reveal that bidding is higher in the monetary auction treatment, especially by those with higher CRT scores. In the time auction, there is no clear bidding pattern and there seems to be less of a difference in bidding between individuals with high vs. low CRT scores.
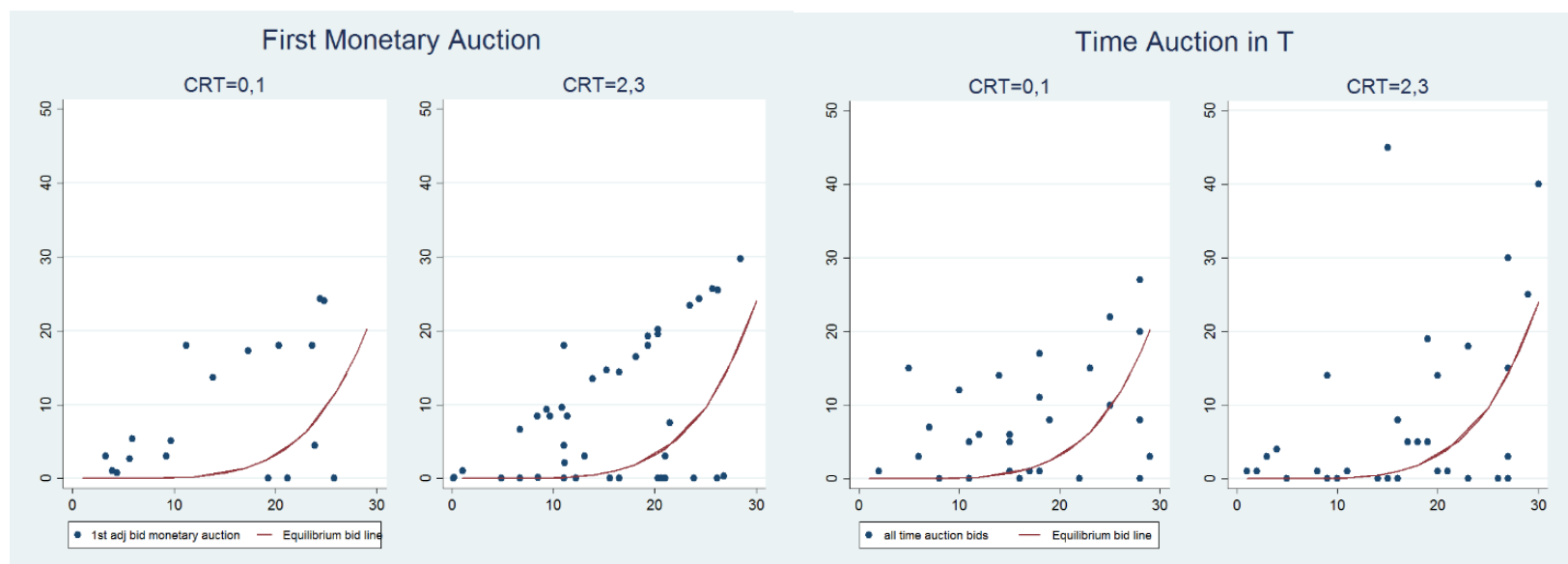

Figure 7. Bidding behavior in the monetary auction vs time auction (T protocol) for CRT types (A) $\mathrm{CRT}=0,1$; (B) $\mathrm{CRT}=2,3$

A Mann-Whitney test provides evidence that there is a significant difference in terms of bidding levels, between bidders' behavior in the time auction played within Protocol MT compared to Protocol T (Prob $>|z|=0.023, n=119$, MWU). On the other hand, if we test for differences in deviations from equilibrium, we find that overbidding in time auctions is similar in $\mathrm{T}$ and MT, both in relative and absolute terms. ${ }^{20}$

Our comparison between money and time is summarized in result 3 below:

Result 3: All-pay auctions for both money and time are characterized by greater than equilibrium bidding on average and negative bidder payoffs. There is no significant difference in the incidence or the extent of overbidding between auctions for money or time. Auctions for time are less efficient than those for money. While measures of player

\footnotetext{
${ }^{20}$ Relative to the equilibrium, subjects overbid on average 1.38 in MT compared with 3.25 in $\mathrm{T}$, not significantly different ( $p=0.243, N 1=59, N 2=60, t$-test). In terms of absolute deviations from the Bayesian Equilibrium the difference is also not significant: 5.32 in treatment MT compared with 6.65 in treatment $\mathrm{T}(p=0.28, N 1=59, N 2=60$, $t$-test).
} 
sophistication are positively correlated with bidding closer to equilibrium and greater earnings in money auctions, these correlations are not present in auctions for time.

\section{Discussion}

Overbidding in all-pay auctions is a pervasive phenomenon that generalizes from money to an alternative reward medium, time. The aggregate patterns of behavior are similar in our auctions played for money and those played for time. The rent available from winning the auction is more than dissipated in both auctions. Thus, the overdisspation of rent is likely to occur not just when competition is in terms of expenditures, but when it is in terms of time. Efficiency, whether bidding for money or time, is low compared to the levels typically observed in winner-pay auctions, and this suggests that it may not be a good idea for economists interested in achieving high levels of efficiency to recommend competing for a prize. This is because of the heterogeneity in behavior of individuals when they are engaging in a competition. In our auctions, some individuals, our zero bidders, choose not to compete. Others, our diagonal bidders, compete too aggressively. The result is that in many cases individuals with lower valuations, with a tendency to bid aggressively, will sometimes win the auction at the expense of a low-bidding player with a higher valuation.

The overall similarity in terms of overbidding behavior between the auctions for money and time conceals a number of important differences at the level of individual behavior. A gender effect in behavior exists in the time auction though not in the money auctions, with women bidding significantly higher than men in the time auction. Cognitive sophistication and accurate calibration of probabilities is strongly related to bidding behavior and performance in the money auctions, but these relationships do not appear in the time auction. This pattern may be due to a correlation between cognitive sophistication and the relative weight placed on money relative to time. More sophisticated individuals may apply their attention more to the setting in which better decisions translate into more money rather than more time.

Typically, results from the student subjects that participate in laboratory experiments generalize well to other populations. However, students may have a relatively low cost of time compared to the value of money so that the stakes may differ between the time and money auctions. This tradeoff might be different for other populations, such as busy professionals with high incomes, for whom time is very scarce relative to money. Thus, future research could productively consider whether behavior in time auctions is similar for different demographic groups. 
Previous work, beginning with Niederle and Vesterlund (2007) would have led us to believe that men would bid more than women because of their higher level of competitiveness. In our time auctions, we observe the opposite; that men bid lower than women. Although the all pay auction is thought of as a model of competition in economic theory, it may not be viewed as such by our participants.

In the all-pay monetary auction, there is a strong relationship between measures of sophistication, the CRT and probability calibration scores, and bidding behavior. Individuals with higher CRT scores and better calibration are less subject to overbidding and earn more. In future research, it would be interesting to group sophisticated people together, make it common knowledge that all bidders are sophisticated, and to study how closely behavior conforms to the Bayesian equilibrium. Such an approach has been taken by Bosch-Rosa et al. (2017) for markets for long lived assets, and they observe that prices conform much more closely to fundamental values when agents are more sophisticated. The relationships between sophistication and behavior disappear in the time auction. Bidding behavior in the time auction may have correlates that we have not measured in this study. This possibility can be explored in future work.

Another area for follow up work would consider whether the results are similar in terms of real effort. Competing in contests may involve money and time, but may also involve costly effort. A future research project could consider whether a contest in which a real effort task is conducted with the winner receiving a prize behaves similarly to the auctions that we have studied here.

\section{References}

Abdellaoui, M., Vossmann, F., and Weber, M. (2005). Choice-Based Elicitation and Decomposition of Decision Weights for Gains and Losses under Uncertainty. Management Science, 51, (9), 1384-1399.

Andersen, S., Fountain J., Harrison G., and Rutstroem E. (2014). Estimating Subjective Probabilities, Journal of Risk and Uncertainty 48(3), 207 - 229.

Andreoni, J. (1995). Cooperation in Public-Goods Experiments: Kindness or Confusion? The American Economic Review. 85 (4), 891-904.

Armantier O., Treich N., (2009). Subjective probabilities in games: an application to the overbidding puzzle. International Economic Review. 50 (4), 1079-1102.

Artinger F., Exadaktylos F., Koppel H., Sääksvuori L. (2010), Applying quadratic scoring rule transparently in multiple choice settings: a note, Jena Economic Research Papers, 2010-2021 
Baharad E, Nitzan S (2008) Contest efforts in light of behavioral considerations. The Economic Journal 118, (533) 2047-2059.

Barut, Y., Kovenock, D., and Noussair, C., (2002). A comparison of multiple-unit all-pay and winner-pay auctions under incomplete information. International Economic Review 43, 675-707.

Berger, R., Rauhut, H., Prade, S., and Helbing, D. (2012). Bargaining over time in ultimatum game experiments. Social Science Research, 41 (2), 372-379.

Blanco, M., Engelmann, D., Koch, A. K., and Normann, H.-T. (2010). Belief elicitation in experiments: is there a hedging problem? Experimental Economics 13(4), 412 - 438.

Bosch-Rosa, C., Meissner, T and Bosch-Domenech, A. (2017) Cognitive Bubbles. Experimental economics, forthcoming.

Brier, Glenn W. (1950). Verification of Forecasts Expressed in Terms of Probability. Monthly

Weather Review 78, 1-3.

Chen Y., Katuščák P., Ozdenoren E. (2013), Why can't a woman bid more like a man?, Games and Economic Behavior, 77 (1),181-213.

Cox, J., Smith, V., Walker, J., (1988). Theory and individual behavior of first price auctions. Journal of Risk and Uncertainty 1, 61-99.

Davis, D., Reilly, R., (1998). Do too many cooks always spoil the stew? An experimental analysis of rentseeking and the role of a strategic buyer. Public Choice 95, 89-115.

Dechenaux, E., Kovenock, D. and Sheremeta, R.M. (2012) A survey of experimental research on contests, all-pay auctions and tournaments. Chapman University, ESI Working Paper.

Delgado M., Schotter A., Ozbay E., and Phelps E. (2008), Understanding Overbidding: Using the Neural Circuitry of Reward to Design Economic Auctions, Science 26, 321 (5897), 1849-1852.

Ernst, C. \& Thöni, C. (2009). Bimodal Bidding in Experimental All-Pay Auctions. University St. Galen, Working Paper.

Frederick, S., 2005. Cognitive reflection and decision making. Journal of Economic Perspectives 19, 2542.

Gächter S., Renner E.. (2010). The effects of (incentivized) belief elicitation in public goods experiments. Experimental Economics. 13 (3), 364-377.

Gneezy, U., Smorodinsky, R. (2006), All-pay auctions: an experimental study. Journal of Economic Behavior and Organization, 61 (2), 255-275.

Goeree J., Holt C., Palfrey T. (2002), Quantal response equilibrium and overbidding in private-value auctions, Journal of Economic Theory, 104 (1), 247-272.

Ham J. C., Kagel J. H.(2006), Gender effects in private value auctions, Economics Letters, 92 (3), 375-382.

Hyndman K., Erkut Y. Ozbay, Sujarittanonta P. (2012), Rent seeking with regretful agents: Theory and experiment, Journal of Economic Behavior \& Organization, 84 (3), 866-878. 
Jones, G., 2008. Are smarter groups more cooperative? Evidence from prisoner's dilemma experiments 1959-2003. Journal of Economic Behavior and Organization 68, 489-497.

Kadane, J. B., and Winkler R. L. (1988). Separating probability elicitation from utilities. Journal of the American Statistical Association 83 (402), 357-363.

Kaplan, T.R., Luski, I., Sela, A., and Wettstein, D. (2002), All-pay auctions with variable rewards. Journal of Industrial Economics 50, (4), 417-430.

Ku, G., Malhotra, D., Murnighan, J.K., 2005. Towards a competitive arousal model of decision making: A study of auction fever in live and Internet auctions. Organizational Behavior and Human Decision Processes 96 (2), 89-103

Liu, T.X., Yang, J., Adamic, L.A. and Chen, Y. (2011). Crowdsourcing with all-pay auctions: a field experiment on Taskcn. Proceedings of the American Society for Information Science and Technology 48, (1) , 1-4

Lugovskyy, V., Puzzello, D. \& Tucker, S. (2010). An experimental investigation of overdissipation in the all-pay auction. European Economic Review, 54, 974-997.

Millet, K., Dewitte, S., (2006). Altruistic behavior as a costly signal of general intelligence. Journal of Research in Personality 41, 316-326.

Müller, W., Schotter, A. (2010). Workaholics and Dropouts in Organizations. Journal of the European Economic Association, 8, 717-743.

Murphy K., Shleifer A. and Vishny R. W. (1993). Why Is Rent-Seeking So Costly to Growth? The American Economic Review. 83 (2), 409-414.

Noussair C.N., Stoop J. (2015). Time as a Medium of Reward in Three Social Preference Experiments, Experimental Economics 18(3), 442 - 456.

Noussair C., Silver J. (2006), Behavior in all-pay auctions with incomplete information, Games and Economic Behavior, 55 (1), 189-206.

Nyarko Y., Schotter A. (2002). An Experimental Study of Belief Learning Using Elicited Beliefs. Econometrica 70, (3), 971-1005.

Offerman, T., Sonnemans, J., Schram, A., (1996). Value orientations, expectations, and voluntary contributions in public goods, Economic Journal, 106, 817-845.

Offerman, T., Sonnemans J., van de Kuilen G., Wakker P. (2009). A Truth-Serum for Non-Bayesians: Correcting Proper Scoring Rules for Risk Attitudes. Review of Economic Studies, 76, (4), 1461-1489.

Potters, J.C., De Vries, C.G. \& Van Winden, F. (1998). An Experimental Examination of Rational Rent Seeking. European Journal of Political Economy, 14, 783-800.

Rey-Biel, P. (2009). Equilibrium play and best response to (stated) beliefs in normal form games. Games and Economic Behavior, 65 (2), 572-585.

Rubalcava L, Teruel G, Thomas D. (2009) Investments, time preferences and public transfers paid to women. Economic Development and Cultural Change 57(3):507-538, doi:10.1086/596617. 
Sheremeta, R. M.. (2013).Overbidding and heterogeneous behavior in contest experiments, Journal of Economic Surveys. 27 (3), 491-514.

Schneider, M., \& Porter, D. (2016). Cognitive Reflection Predicts Decision Quality in Individual and Strategic Decisions. ESI Working Paper (No. 16-24).

Schotter, A. and B. Sopher. (2006). Trust and trustworthiness in intergenerational games: An experimental study of inter-generational advice. Experimental Economics 9(2), 123-145.

Starmer, C. (2000). Developments in Non-Expected Utility Theory: The Hunt for a Descriptive Theory of Choice under Risk. Journal of Economic Literature, 38(2), 332-382.

Trautmann S., Kuilen G.. (2014). Belief Elicitation: A Horse Race among Truth Serums, Economic Journal 125(589), $2116-2135$.

Zizzo, D. (2010). Experimenter demand effects in economic experiments. Experimental Economics, 13, 75 98. 


\section{ONLINE APPENDIX}

\section{Appendix A, Bayesian equilibrium for the all-pay auctions}

This appendix contains a more detailed derivation of a risk neutral Bayesian equilibrium of the monetary all-pay auction. The derivation reproduces the argument presented by Noussair and Silver (2006).

Suppose that all bidders draw a valuation $v_{i}$ from a common distribution $F(v)$, In an all-pay auction, they follow a common equilibrium bidding strategy, $\beta_{i}\left(v_{i}\right)=\beta(v)$, for all $i$. We assume that the strategy is symmetric and strictly monotonic and therefore invertible. Let $\beta^{-1}(b)$ be the inverse of $\beta(v)$. The probability that bidder $i$, with a bid of $b_{i}$, wins the auction, is equal to the probability that none of the $n-1$ participants make higher bids. Therefore, bidder $i$ 's expected payoff can be written as is:

$$
\Pi_{i}\left(b_{i}, v_{i}\right)=v_{i} * F^{n-1}\left(\beta^{-1}\left(b_{i}\right)\right)-b_{i}
$$

As $v_{i}$ is distributed uniformly in the experiment on $[0, \bar{v}]$, for the experimental environment we have that

$$
F\left(\beta^{-1}(b)\right)=\beta^{-1}(b) / \bar{v},
$$

The expected payoff then becomes:

$$
\Pi_{i}\left(b_{i}, v_{i}\right)=v_{i} *\left(\frac{\beta^{-1}\left(b_{i}\right)}{\bar{v}}\right)^{n-1}-b_{i}
$$

We solve the corresponding maximization problem:

$\frac{\partial \Pi_{i}(b, v)}{\partial b_{i}}=0 \Leftrightarrow(n-1) * \frac{v_{i}}{\bar{v}} *\left(\frac{\beta^{-1}\left(b_{i}\right)}{\bar{v}}\right)^{n-2} * \frac{d \beta^{-1}\left(b_{i}\right)}{d b_{i}}-1=0$

Since $\beta\left(v_{i}\right)=b_{i}$, we have that $\beta^{-1}\left(b_{i}\right)=v_{i}$, and $\frac{d \beta^{-1}\left(b_{i}\right)}{d b_{i}}=\frac{1}{\beta^{\prime}\left(v_{i}\right)}$. Therefore,

$\beta^{\prime}\left(v_{i}\right)=(n-1) *\left(\frac{v_{i}}{\bar{v}}\right)^{n-1}$

In order to solve for the Bayesian equilibrium, we integrate the expression in (A5).

$\beta\left(v_{i}\right)=\int_{0}^{v_{i}} \beta_{i}^{\prime}\left(v_{i}\right) d v_{i}=\int_{0}^{v_{i}}(n-1) *\left(\frac{v_{i}}{\bar{v}}\right)^{n-1} d v_{i}=\left(\frac{1}{\bar{v}}\right)^{n-1} * \frac{n-1}{n} * v_{i}^{n}+C$

Since it can be assumed that $\beta(0)=0$, we set $C=0$. It follows that the equilibrium bidding function must satisfy:

$$
\beta\left(v_{i}\right)=\left(\frac{1}{\bar{v}}\right)^{n-1} * \frac{n-1}{n} * v_{i}^{n}
$$

The expected payoff of bidder $i$ is then: 


$$
\begin{aligned}
& \Pi_{i}\left(b_{i}, v_{i,}\right)=v_{i} *\left(\frac{\beta^{-1}(b i)}{\bar{v}}\right)^{n-1}-b_{i} \Leftrightarrow \\
& \Pi_{i}\left(v_{i}\right)=v_{i} *\left(\frac{v_{i}}{\bar{v}}\right)^{n-1}-\beta\left(v_{i}\right) \Leftrightarrow \Pi\left(v_{i}\right)_{i}=v_{i} *\left(\frac{v_{i}}{\bar{v}}\right)^{n-1}-\left(\frac{1}{\bar{v}}\right)^{n-1} * \frac{n-1}{n} * v_{i}^{n} \Leftrightarrow \\
& \Pi_{i}\left(v_{i}\right)=\left(\frac{1}{\bar{v}}\right)^{n-1} * v_{i}^{n} * \frac{1}{n}
\end{aligned}
$$

\section{Appendix B: Instructions}

\section{Instructions Protocol MT:}

ID:

Welcome everybody!

You are about to participate in an experiment in the economics of decision-making. For participating in this experiment, you will receive a show up fee of $10 €$. Any extra earnings gained during the experiment will be added to this sum. The total earnings will be paid by bank transfer within the next $48 \mathrm{~h}$. The experiment will consist of three parts. In the first part you will be required to answer a few questions. The second part will consist of series of auctions in which you will be bidding for a unit of good called "Y". In part 3 you will play a game that will determine the time when you can leave the experiment.

After each stage, we will distribute the instructions for the following stage. Also, any form of communication with other participants is prohibited. Any questions should be directed towards the instructors. Any deviation of this rule will result in direct exclusion from the experiment and loss of any return. Please write on every page received your ANR in the left top corner of your documents.

\section{Stage 1:}

Please answer the following questions as good as you can.

Each question you answer correctly in this stage will increase your total earnings by $\mathbf{1} €$.

QUESTION NR. 1: 
A shirt and a hat cost $5.50 €$ in total. The shirt costs $5 €$ more than the hat. How much does the hat costs? cents

\section{QUESTION NR. 2:}

If it takes 3 people 3 hours to assemble 3 bicycles, how long would it take 90 people to assemble 90 bicycles? hours

\section{QUESTION NR. 3:}

The forest of North Carolina is home to a colony of wild rabbits. Every year, the colony doubles in size. If it takes 24 years for the colony to reach 100.000 rabbits, how long would it take for the colony to reach half of that number? years

\section{ID:}

\section{Stage 2:}

In the current stage you will take part in multiple auctions. There will be held 15 auctions and 1 practice period. Each period you will be grouped with other 4 participants. Identifying the participants in your group is not possible. The currency used within the experiment is called "ECU". The conversion rate is 100 ECU to 1 euro. In the beginning you will receive an initial endowment of 1000 ECU.

In each period, a single unit, called "Y", will be auctioned separately to each group. A Record sheet will be provided. On the Record Sheet, under the column "Product value", a value is written for each period. This value represents your product valuation and is an indication of the amount of ECU you can obtain if you win unit "Y". The "Product Value" is chosen randomly before the experiment and independently from other player's valuation and will be an integer between 1 and 1000. Every period you can submit a positive bid by filling in the amount in column "Bid". You will pay the bid whether you win or not. All participants will submit their bids simultaneously without having any information about the bids of other subjects. The bidder with the highest bid, within each group, will receive unit "Y". In case of a tie, the unit will be randomly assigned by a coin toss.

\section{Calculating your earning:}


In case that you have the highest bid within the auction, you have to write under the column "ECU won" your "Product Value" for that period; otherwise a 0 should be filled in. After every auction, you have to record your earnings, which you can easily calculate by subtracting Column 3 from Column 4, in the column named "Earnings per period".

At the end of the experiment, your total return will be the sum of all your period earnings plus the initial endowment of $1000 \mathrm{ECU}$.

Example : Suppose that your Product value is 300 and you write a bid of 250 in the column "Bid". If you win the auction by having the highest bid among all bids, you will write 300 under "ECU won". Therefore, your earnings for that period will be equal to $300-250=50$. If you happen not to win the auction, you will write 0 under "ECU won" and - 250 under "Earnings per period".

\section{ID:}

\section{Reporting probabilities}

Before we start the auctions, you will be given a series of questions in which you will be asked to report the probability with which you believe a person can win the auction, by bidding a certain amount of ECUs. You should report a probability from $0 \%$ to $100 \%$. Your earnings will be determined by the accuracy of your predictions. The reported probability will be compared with the probability of winning the auction in a previous session by placing the specified bid in the question. The reported probabilities for each bid will not influence any of your potential payoffs in the later stages of the experiment. Your potential earnings will be calculated using the following formula:

\section{Payoff $=\max \left\{0,10-45\left(P_{t}--P_{a}\right)^{2}\right\}$}

Basically, what the formula does is that it penalizes you every time your reported probability is different than the actual probability. You can obtain the most revenue when the actual probability is equal to the reported probability. The most you can get is $10 €$. Let's look at an example:

Example: Suppose an individual plays the auction presented above against 4 other people. All players' values are equally likely to be any value between 1 and 1000. What are the chances that the individual wins the auction if he bids 20 ? If you believe that the probability of winning the 
auction is $38 \%$ but the actual probability is $20 \%$, you can receive: Payoff $=\max \{0,10-45(0.38$ $\left.0.20)^{2}\right\}=8.54 €$

At the end of the experiment, one of the periods will be chosen at random to be paid. Depending on how accurate your prediction is for that period, you will receive the amount of Euros corresponding to the formula presented. Therefore, you should respond as accurate as you can in each period, as any of your choices could prove to be the one that determines the payment.

Question 1: Suppose an individual plays the auction presented above against 4 other people. All players' values are equally likely to be any value between 1 and 1000 . What are the chances that the individual wins the auction if he bids $\mathbf{5 0}$ ? $\%$

Question 2: Suppose an individual plays the auction presented above against 4 other people. All players' values are equally likely to be any value between 1 and 1000 . What are the chances that the individual wins the auction if he bids $\mathbf{1 0 0}$ ? $\%$

Question 3: Suppose an individual plays the auction presented above against 4 other people. All players' values are equally likely to be any value between 1 and 1000. What are the chances that the individual wins the auction if he bids $\mathbf{1 5 0}$ ? $\%$

Question 4: Suppose an individual plays the auction presented above against 4 other people. All players' values are equally likely to be any value between 1 and 1000. What are the chances that the individual wins the auction if he bids $\mathbf{2 0 0}$ ? $\%$

Question 5: Suppose an individual plays the auction presented above against 4 other people. All players' values are equally likely to be any value between 1 and 1000 . What are the chances that the individual wins the auction if he bids $\mathbf{2 5 0}$ ? $\%$

Question 6: Suppose an individual plays the auction presented above against 4 other people. All players' values are equally likely to be any value between 1 and 1000 . What are the chances that the individual wins the auction if he bids $\mathbf{3 0 0}$ ? $\%$

Question 7: Suppose an individual plays the auction presented above against 4 other people. All players' values are equally likely to be any value between 1 and 1000. What are the chances that the individual wins the auction if he bids $\mathbf{3 5 0}$ ? $\%$ 
Question 8: Suppose an individual plays the auction presented above against 4 other people. All players' values are equally likely to be any value between 1 and 1000 . What are the chances that the individual wins the auction if he bids $\mathbf{4 0 0}$ ? $\%$

Question 9: Suppose an individual plays the auction presented above against 4 other people. All players' values are equally likely to be any value between 1 and 1000 . What are the chances that the individual wins the auction if he bids $\mathbf{4 5 0}$ ? $\%$

Question 10: Suppose an individual plays the auction presented above against 4 other people. All players' values are equally likely to be any value between 1 and 1000. What are the chances that the individual wins the auction if he bids $\mathbf{5 0 0}$ ? $\%$

Question 11: Suppose an individual plays the auction presented above against 4 other people. All players' values are equally likely to be any value between 1 and 1000. What are the chances that the individual wins the auction if he bids $\mathbf{5 5 0}$ ? $\%$

Question 12: Suppose an individual plays the auction presented above against 4 other people. All players' values are equally likely to be any value between 1 and 1000. What are the chances that the individual wins the auction if he bids $\mathbf{6 0 0}$ ? $\%$

Question 13: Suppose an individual plays the auction presented above against 4 other people. All players' values are equally likely to be any value between 1 and 1000. What are the chances that the individual wins the auction if he bids $\mathbf{6 5 0}$ ? $\%$

Question 14: Suppose an individual plays the auction presented above against 4 other people. All players' values are equally likely to be any value between 1 and 1000 . What are the chances that the individual wins the auction if he bids $\mathbf{7 0 0}$ ? $\%$

Question 15: Suppose an individual plays the auction presented above against 4 other people. All players' values are equally likely to be any value between 1 and 1000 . What are the chances that the individual wins the auction if he bids $\mathbf{7 5 0}$ ? $\%$

Question 16: Suppose an individual plays the auction presented above against 4 other people. All players' values are equally likely to be any value between 1 and 1000 . What are the chances that the individual wins the auction if he bids $\mathbf{8 0 0}$ ? $\%$ 
Question 17: Suppose an individual plays the auction presented above against 4 other people. All players' values are equally likely to be any value between 1 and 1000. What are the chances that the individual wins the auction if he bids $\mathbf{8 5 0}$ ? $\%$

Question 18: Suppose an individual plays the auction presented above against 4 other people. All players' values are equally likely to be any value between 1 and 1000 . What are the chances that the individual wins the auction if he bids $\mathbf{9 0 0}$ ? $\%$

Question 19: Suppose an individual plays the auction presented above against 4 other people. All players' values are equally likely to be any value between 1 and 1000 . What are the chances that the individual wins the auction if he bids $\mathbf{9 5 0}$ ? $\%$

\section{ID :}

Now that you have assessed the probabilities we can start the auctions. Firstly, we will have one period for practice purpose. Please take Record Sheet Nr. 1 and fill in you bid in the practice period. Your Endowment is 1000 ECU. You are not allowed to fill in your bids before each stage ends.

\section{Record sheet Nr. 1}

\begin{tabular}{|l|l|l|l|l|}
\hline \multicolumn{1}{|c|}{ Period } & Product Value & Bid & ECU won & Earnings per period \\
\hline Practice & 425 & & & \\
\hline 1 & 422 & & & \\
\hline 2 & 273 & & & \\
\hline 3 & 181 & & & \\
\hline 4 & 36 & & & \\
\hline 5 & 276 & & & \\
\hline 6 & 855 & & & \\
\hline 7 & 651 & & & \\
\hline 8 & 860 & & & \\
\hline 9 & 676 & & & \\
\hline 10 & 197 & & & \\
\hline 11 & 331 & & & \\
\hline 12 & 158 & & & \\
\hline 13 & 110 & & & \\
\hline 14 & 658 & & & \\
\hline 15 & 998 & & & \\
\hline
\end{tabular}




$$
\text { period }=\_ \text {ECU }{ }^{\text {Total earnings per }}
$$

Total Earnings $=1000+$ ECU

\section{ID:}

\section{$\underline{\text { Stage } 3}$}

In this stage, everybody must wait $\mathbf{3 0}$ minutes in the laboratory. For taking part in stage 3 you receive an additional one-time payment of $\mathbf{6} €$. This extra payment is independent of your show-up fee of $10 €$. During the mandatory waiting time, you are not allowed to do anything. You cannot use the computer or any other device, you cannot read and you cannot communicate with other participants. However, you can have the chance to decrease the mandatory time and leave the laboratory earlier by participating in an auction similar to the one in stage 2. A paper called "Record Sheet Nr. 2" will be provided. The instructions of the auction are similar to the one in stage 2 , with some exceptions. There will be no endowment given at the beginning of the auction. Each participant receives an unique "Time exemption" which is chosen randomly and independently before the experiment and is any integer between 1 and 30 . This value is recorded under the column "Time exemption". The currency used within the auction is time. Therefore, all the biddings and payoffs will be made using minutes. As before, you will be grouped randomly with other 4 participants. Every bid submitted and written under column "Bid" will be paid. If you are successful in winning the auction (have the highest bid among the 5 players), the number of minutes that you win after you pay your bid, will be subtracted from the mandatory waiting time and you can leave the laboratory earlier. If you are unfortunate and loose the auction, your waiting time will not be decreased. However, you will have to pay your bid by staying on top of the mandatory time, the number of minutes you bided. Please read carefully the following examples. Afterwards, a practice period will be held.

Example 1: Let's suppose that you're "Time exemption" is 12 minutes. If you bid 14 minutes and your bid is the highest among all the other bidders, then after paying the bid $(12-14=-2)$ you will actually have to wait in the laboratory an additional 2 minutes after stage 3 is over. 
Example 2: Suppose that you're "Time exemption" is 20 minutes and you bid an amount of 11 minutes and you win the auction. Then, you will be able to decrease your mandatory waiting time by 9 minutes. $(20-11=9)$. In other words, you will be able to leave the experiment 9 minutes earlier than when the experiment is supposed to finish.

Example 3: Suppose that you have a "Time exemption" equal to 12 and you bid 6 minutes.

However, you are not successful in winning the auction. Therefore, you will have to wait in the laboratory an additional 6 minutes after stage 3 is over.

\section{ID participant:}

\section{Record sheet Nr. 2}

You are not allowed to fill in your bids before each stage ends.

Practice period: Time exemption $=$

Bid

Total waiting time

Period 1: Time exemption $=$

Bid

Total waiting time

After finishing the auction please fill in some questions regarding your background. The answers will remain confidential as you will provide only your ID.

After this, the experimenter will announce when the mandatory 30 minutes period starts. Then, you have to wait patiently in your chair, until the total amount of waiting time which you have to remain in laboratory (adjusted by the minutes won or lost in the auction), elapses.

Thank you for your cooperation!

\section{Instructions Protocol T:}


ID:

Welcome everybody!

You are about to participate in an experiment in the economics of decision-making. For participating in all 2 stages experiment, you will receive a fixed endowment of $5 €$. Any extra earnings gained during the experiment will be added to this sum. The total earnings will be paid by bank transfer within the next $24 \mathrm{~h}$. The experiment will consist of two parts. In the first part you will be required to answer a few questions. Each question responded correctly will bring you $1 €$. The second part will consist of an auction and a game.

Written in the left top corner of your instruction sheet, you will find an unique ID. Please turn the page in order to begin stage 1 .

\section{Stage 1:}

Please answer the following questions as good as you can.

Each question you answer correctly will increase your total earnings by $1 €$. QUESTION NR. 1:

A shirt and a hat cost $5.50 €$ in total. The shirt costs $5 €$ more than the hat. How much does the hat costs? cents

QUESTION NR. 2:

If it takes 3 people 3 hours to assembly 3 bicycles, how long would it take 90 people to assembly 90 bicycles? hours

\section{QUESTION NR. 3:}

The forest of North Carolina is home to a colony of wild rabbits. Every year, the colony doubles in size. If it takes 24 years for the colony to reach 100.000 rabbits, how long would it take for the colony to reach half of that number? years

\section{Stage 2}

In this stage, everybody must wait $\mathbf{3 0}$ minutes in the laboratory. During the mandatory waiting time, you are not allowed to do anything. You cannot use the computer or any other device, you cannot read and you cannot communicate with other participants. However, you can have the 
chance to decrease the mandatory time and leave the laboratory earlier by participating in an auction.

The "Time Redemption" chosen randomly and independently before the experiment, is likely any integer between 1 and 30 and is recorded under the column " Time Redemption". The currency used within the auction is time. Therefore, all the biddings and payoffs will be made using minutes.

You will be grouped randomly with other 4 participants. Identifying the participants in your group is not possible. Please record your bid and results in stage 2, on the Record Sheet Nr. 1 provided in the beginning of the experiment. Every period you can submit a positive bid by filling in the amount in column "Bid". You will pay the bid whether you win or not. The bidder with the highest bid, within each group, will win the auction. In case of a tie, the unit will be randomly assigned by a coin toss.

If you are successful in winning the auction, the number of minutes that you win after you pay your bid, will be subtracted from the mandatory waiting time and you can leave the laboratory earlier. If you are unfortunate and loose the auction, your waiting time will not be decreased. However, you will have to pay your bid by staying on top of the mandatory time, the number of minutes you bided.

Firstly, a practice period will be held.

Example 1: Let's suppose that you're "Time exemption" is 12 minutes. If you bid 14 minutes and your bid is the highest among all the other bidders, then after paying the bid (12-14= -2$)$ you will actually have to wait in the laboratory an additional 2 minutes after stage 3 is over.

Example 2: Suppose that you're "Time exemption" is 20 minutes and you bid an amount of 11 minutes and you win the auction. Then, you will be able to decrease your mandatory waiting time by 9 minutes. $(20-11=9)$. In other words, you will be able to leave the experiment 9 minutes earlier than when the experiment is supposed to finish.

Example 3: Suppose that you have a "Time exemption" equal to 12 and you bid 6 minutes. However, you are not successful in winning the auction. Therefore, you will have to wait in the laboratory an additional 6 minutes after stage 3 is over. 


\section{Reporting Probabilities}

Before we start the auction, you will be given a series of questions in which you will be asked to report the probability with which you believe a person can win the auction, by bidding a certain amount minutes. You should report a probability from $0 \%$ to $100 \%$. Your earnings will be determined by how accurate your prediction will be. The reported probability will be compared with the average probability of winning the auction in a previous session from a randomly chosen group. The reported probabilities for each bid will not influence any of your potential payoffs in the later stages of the experiment. Your potential earnings will be calculated using the following formula: Payoff $=\max \left\{0,10-45\left(\mathbf{P}_{t}--P_{a}\right)^{2}\right\}$

Basically, what the formula does is that it penalizes you every time your reported probability is different than the actual probability. You can obtain the most revenue when the actual probability is equal to the reported probability. The most you can get is $10 €$. Let's look at an example:

Example: Suppose an individual plays the auction presented above against 4 other people. All players' values are equally likely to be any value between 1 and 30 . What are the chances that the individual wins the auction if he bids 5? If you believe that the probability of winning the auction is $38 \%$ but the actual probability is $20 \%$, you can receive $=$ Payoff $=\max \left\{0,10-45(0.38-0.20)^{2}\right.$ \}$=8.54 €$

At the end of the experiment, one of the periods will be chosen at random to be paid. Depending on how accurate your prediction is for that period, you will receive the amount of ECU corresponding to the Decision Table presented. Therefore, you should respond as accurate as you can in each period, as any of your choices could prove to be the one that determines the payment.

Question 1: Suppose an individual plays the auction presented above against 4 other people. All players' values are equally likely to be any value between 1 and 30 . What are the chances that the individual wins the auction if he bids 2 ? $\%$ 
Question 2: Suppose an individual plays the auction presented above against 4 other people. All players' values are equally likely to be any value between 1 and 30 . What are the chances that the individual wins the auction if he bids 4 ? $\%$

Question 3: Suppose an individual plays the auction presented above against 4 other people. All players' values are equally likely to be any value between 1 and 30 . What are the chances that the individual wins the auction if he bids 6 ? $\%$

Question 4: Suppose an individual plays the auction presented above against 4 other people. All players' values are equally likely to be any value between 1 and 30 . What are the chances that the individual wins the auction if he bids $\mathbf{8}$ ? $\%$

Question 5: Suppose an individual plays the auction presented above against 4 other people. All players' values are equally likely to be any value between 1 and 30 . What are the chances that the individual wins the auction if he bids $\mathbf{1 0}$ ? $\%$

Question 6: Suppose an individual plays the auction presented above against 4 other people. All players' values are equally likely to be any value between 1 and 30 . What are the chances that the individual wins the auction if he bids $\mathbf{1 2}$ ? $\%$

Question 7: Suppose an individual plays the auction presented above against 4 other people. All players' values are equally likely to be any value between 1 and 30 . What are the chances that the individual wins the auction if he bids 14 ? $\%$

Question 8: Suppose an individual plays the auction presented above against 4 other people. All players' values are equally likely to be any value between 1 and 30 . What are the chances that the individual wins the auction if he bids $\mathbf{1 6}$ ? $\%$

Question 9: Suppose an individual plays the auction presented above against 4 other people. All players' values are equally likely to be any value between 1 and 30 . What are the chances that the individual wins the auction if he bids $\mathbf{1 8}$ ? $\%$ 
Question 10: Suppose an individual plays the auction presented above against 4 other people. All players' values are equally likely to be any value between 1 and 30 . What are the chances that the individual wins the auction if he bids $\mathbf{2 0}$ ? $\%$

Question 11: Suppose an individual plays the auction presented above against 4 other people. All players' values are equally likely to be any value between 1 and 30 . What are the chances that the individual wins the auction if he bids 22 ? $\%$

Question 12: Suppose an individual plays the auction presented above against 4 other people. All players' values are equally likely to be any value between 1 and 30 . What are the chances that the individual wins the auction if he bids 24 ? $\%$

Question 13: Suppose an individual plays the auction presented above against 4 other people. All players' values are equally likely to be any value between 1 and 30 . What are the chances that the individual wins the auction if he bids $\mathbf{2 6}$ ? $\%$

Question 14: Suppose an individual plays the auction presented above against 4 other people. All players' values are equally likely to be any value between 1 and 30 . What are the chances that the individual wins the auction if he bids $\mathbf{2 8}$ ? $\%$

\section{Auction:}

Now that you have assessed the probabilities we can start the auctions. Firstly, we will have one period for practice purpose. Please take Record Sheet Nr. 1 and fill in the values in the practice period.

\section{ID participant:}

\section{$\underline{\text { Record sheet Nr. } 1}$}

Practice period: Time exemption $=$

Bid

Total waiting time 
Period 1: Time exemption $=$

Bid

Total waiting time

After finishing the auction please fill in the appropriate numbers in Record sheet Nr. 2 and answer a few questions regarding your background. The answers will remain confidential as you will provide only your ID.

After this, the experimenter will announce when the mandatory 30 minutes period starts. Then, you have to wait patiently in your chair, until the total amount of waiting time which you have to remain in laboratory (adjusted by the minutes won or lost in the auction), elapses.

Thank you for your cooperation!

\section{Background Information:}

ID number:

As your ID number is not known either by the instructor or by others participants, this information will remain anonymously. Please fill in the questionnaire or select the appropriate category.

1. What is your age? __ years

2. What is your gender?

(Female / Male)

3. What is your race?

(White, Black, Yellow, Red, Brown)

4. What is your religion?

(Christian / Atheist / Muslim / Buddhist / Others)

5. How many years of higher education you have? years

6. How many siblings do you have?

7. What is your family income per year approximately? Euros 
(10.000-15.000); (15.000 -30.000); (30.000-60.000); (60.000-100.000); higher

8. How many years of higher education do your parents have? years

9. Have you ever encountered the first 3 questions asked in Stage 1 of the experiment? Yes $/ \mathrm{No}$

\section{APPENDIX C: Additional Figures and Tables}

Figure C1: The average difference between the bidding strategies and RNBE, by CRT CRT $<2$ Low ability, differences over the last 5 periods
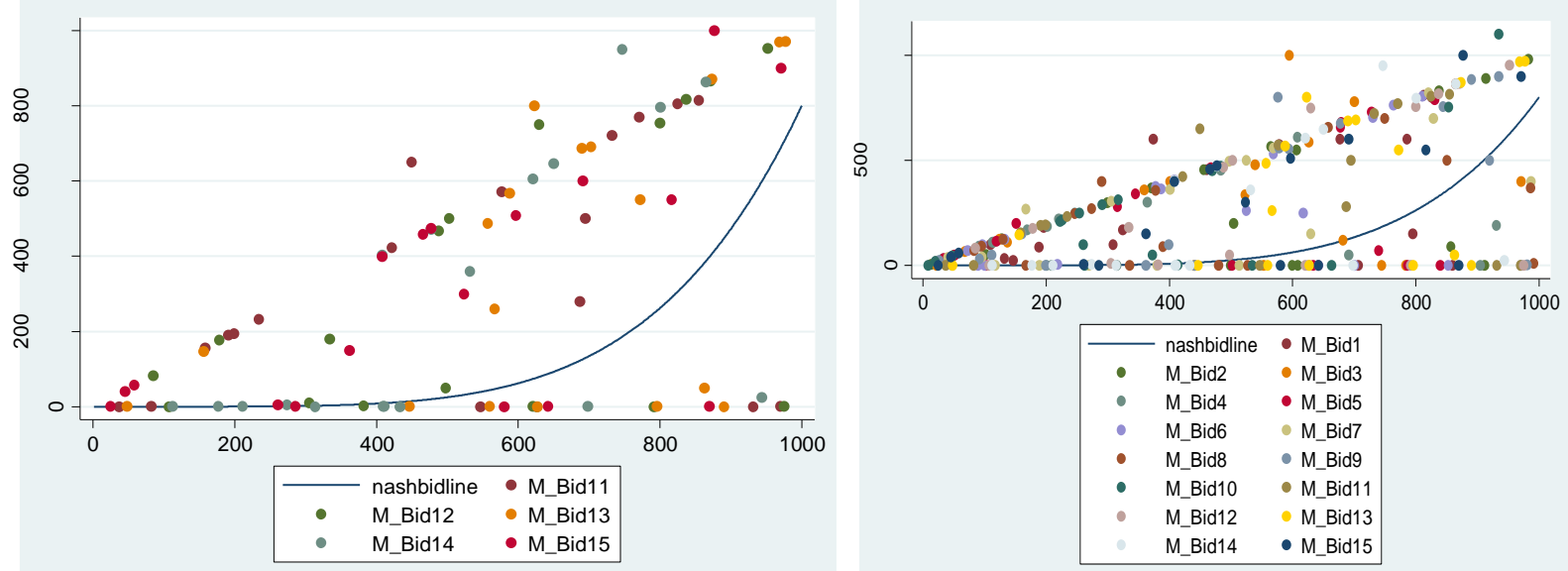

CRT $>2$ High ability, differences (A) over the last 5 periods (B) over all 15 periods
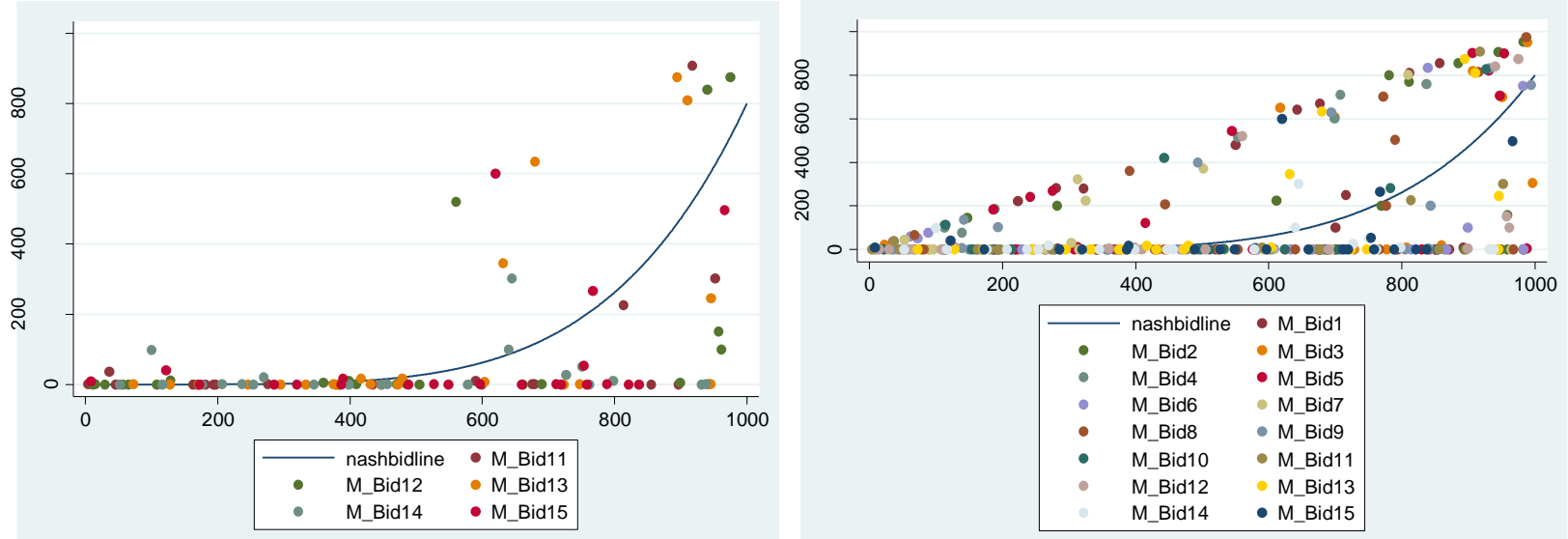
Table C1. OLS Results - Time auction (treatment MT)

(a) Absolute deviation from the RNBE

\begin{tabular}{cccc}
\hline & $(\mathbf{1})$ & $\mathbf{( 3 )}$ & $\mathbf{( 4 )}$ \\
\hline & Abs. Average Bid- & Abs. Average Bid- & Abs. Average \\
& RNBE & RNBE & Bid-RNBE \\
CRT & -1.33 & & -1.15 \\
& $(-1.93)^{*}$ & & $(-1.55)$ \\
Male & & -2.13 & -1.54 \\
& & $(-1.37)$ & $(-0.94)$ \\
Constant & 7.87 & 6.44 & 8.36 \\
& $(5.31)^{* * *}$ & $(6.50)^{* * *}$ & $(5.71)^{* * *}$ \\
Observations & 59 & 58 & 58 \\
Goodness of fit: & $5.8 \%$ & $3.3 \%$ & $7.4 \%$ \\
R-squared & & & \\
\hline
\end{tabular}

Note: dependent variable: Absolute deviation of bid from the risk neutral Bayesian equilibrium in time auction OLS regression, t-value based on robust s.e. in parenthesis; * ${ }^{* *} /{ }^{* * *}$ stands for statistical significance at $10 \%$, $5 \%$ and $1 \%$ level, respectively.

(b) Average earnings

\begin{tabular}{cccc}
\hline & $(\mathbf{1})$ & $\mathbf{( 3 )}$ & $\mathbf{( 4 )}$ \\
\hline & Average & Average & Average \\
& Earnings & Earnings & Earnings \\
CRT & 0.43 & & 0.27 \\
& $(0.54)$ & & $(0.35)$ \\
Male & & 1.58 & 1.44 \\
& & $(1.21)$ & $(1.23)$ \\
Constant & -1.88 & -1.83 & -2.28 \\
& $(-0.98)$ & $(-1.92)^{*}$ & $(-1.13)$ \\
Observations & 59 & 58 & 58 \\
Goodness of fit: & $0 \%$ & $2.5 \%$ & $2.8 \%$ \\
R-squared & & & \\
\hline
\end{tabular}

Note: dependent variable: Average earnings in time auction. OLS regression, coefficient reported, $\mathrm{t}$-value based on robust s.e. in parenthesis; ${ }^{*} / * * * * *$ stands for statistical significance at $10 \%, 5 \%$ and $1 \%$ level, respectively. 
(c) Deviations of bid from the RNBE

\begin{tabular}{cccc}
\hline & $\mathbf{( 1 )}$ & $\mathbf{( 3 )}$ & $\mathbf{( 4 )}$ \\
\hline & Average Bid- & Average Bid-RNBE & Average Bid- \\
& RNBE & & RNBE \\
CRT & -2.44 & & -1.84 \\
& $(-2.42)^{* *}$ & & $(-1.80)^{*}$ \\
Male & & -6.33 & -5.39 \\
& & $(-3.28)^{* * *}$ & $(-2.52)^{* *}$ \\
Constant & 6.05 & 4.468 & 7.52 \\
& $(2.68)^{* * *}$ & $(3.40)^{* * *}$ & $(4.21)^{* *}$ \\
Observations & 59 & 58 & 58 \\
Goodness of fit: & $11 \%$ & $16 \%$ & $22 \%$ \\
R-squared & & & \\
\hline
\end{tabular}

Note: dependent variable: Difference between an individual's bid and the risk neutral Bayesian equilibrium bid OLS regression, t-value based on robust s.e. in parentheses; ${ }^{* * *} /{ }^{* * *}$ stands for statistical significance at $10 \%, 5 \%$ and $1 \%$ level, respectively. 